\title{
Stearoyl-ACP $\Delta^{9}$ Desaturase 6 and 8 (GhA-SAD6 and GhD-SAD8) Are Responsible for Biosynthesis of Palmitoleic Acid Specifically in Developing Endosperm of Upland Cotton Seeds
}

OPEN ACCESS

Edited by:

Junhua Peng,

China National Seed Group Co., Ltd.

China

Reviewed by:

Lingling Zhang,

Wuhan Botanical Garden (CAS),

China

Enrique Martinez Force, Spanish National Research Council

(CSIC), Spain

*Correspondence:

Runzhi Li

rli2001@126.com

Specialty section:

This article was submitted to

Plant Biotechnology,

a section of the journal

Frontiers in Plant Science

Received: 01 March 2019

Accepted: 13 May 2019

Published: 31 May 2019

Citation:

Liu B, Sun Y, Xue J, Mao X, Jia X and Li R (2019) Stearoyl-ACP $\Delta^{9}$ Desaturase 6 and 8 (GhA-SAD6 and GhD-SAD8) Are Responsible for Biosynthesis of Palmitoleic Acid Specifically in Developing Endosperm of Upland Cotton Seeds.

Front. Plant Sci. 10:703. doi: 10.3389/fp/s.2019.00703

\author{
Baoling Liu, Yan Sun, Jinai Xue, Xue Mao, Xiaoyun Jia and Runzhi Li* \\ Institute of Molecular Agriculture and Bioenergy, Shanxi Agricultural University, Jinzhong, China
}

Palmitoleic acid $\left(16: 1 \Delta^{9}\right)$ is one kind of $\omega-7$ fatty acids ( $\omega-7$ FAs) widely used in food, nutraceutical and industry. However, such high-valued $\omega-7$ FA only has a trace level in mature seeds of cotton and other common oil crops. We found that palmitoleic acid (>10.58 Mol\%) was specially enriched in developing cotton endosperm which is disappeared in its mature seed. The present study was conducted to investigate the mechanism underlying high accumulation of palmitoleic acid in developing endosperm but not in embryo of upland cotton (Gossypium hirsutum L.) seed. Of 17 stearoylACP $\Delta^{9}$ desaturases (SAD) gene family members identified in upland cotton, six GhSADs may specifically work in the desaturation of palmitic acid (16:0-ACP) to produce palmitoleic acid (16:1 $\Delta^{9}$-ACP), which were revealed by examining the key amino acids in the catalytic center and their cis-elements. Gene expression analysis showed that spatial patterns of these GhSADs were different in developing ovules, with GhA-SAD6 and $G h D-S A D 8$ preferentially expressed in developing endosperms. Functional analysis by transient expression in Nicotiana benthamiana leaves and genetic complementary assay using yeast mutant BY4389 strain unable to synthesize unsaturated fatty acids demonstrated that GhA-SAD6 and GhD-SAD8 have strong substrate specificity for 16:0-ACP. In contrast, GhA-SAD5 and GhA-SAD7 exhibited high specific activity on 18:0-ACP. Taken together, these data evidence that GhA-SAD6 and GhD-SAD8 are responsible for making palmitoleic acid in developing cotton endosperms, and provide endogenous gene targets for genetic modification to enrich $\omega-7$ FAs in cotton seed oil required for sustainable production of functionality-valued products.

Keywords: cotton, ACP- $\Delta^{9}$ desaturase, palmitoleic acid, endosperm, substrate specificity

\section{INTRODUCTION}

Cotton (Gossypium sp.) is widely cultivated in the world as an important industrial crop, providing excellent fiber and protein plus oil. Particularly, cottonseed oil is an important vegetable oil and broadly used in food/catering, clean biofuel and other industries (Jiao et al., 2013; Sturtevant et al., 2017). Fatty acid composition and its content determine the value and utilization 
of vegetable oil. The conventional cottonseed oil mainly consists of $26 \%$ palmitic acid (16:0), 58\% linoleic acid $\left(18: 2^{\Delta 9,12}\right), 13 \%$ oleic acid $\left(18: 1 \Delta^{9}\right)$ and $2 \%$ stearic acid (18:0) (Zhao et al., 2018). Since linoleic acid is oxidatively unstable, cottonseed oil is easily oxidized and rancid, and thus it normally needs partial hydrogenation before food applications. However, byproducts of this process are trans-fatty acids, which are harmful for human health (Ginter and Simko, 2016). Saturated palmitic acid is not recommended for direct edible use because of its detrimental effects on human health despite of its highly oxidative stability (Shang et al., 2017). Monounsaturated fatty acids (MUFAs) such as oleic acid and palmitoleic acid $\left(16: 1 \Delta^{9}\right)$ are healthpromoting fatty acids, but they are in tiny content in cottonseed oil. Therefore, the development of designer cottonseed oil with nutritionally desirable fatty acids has been an attractive object to meet oil marketing demand.

Omega-7 fatty acids ( $\omega-7$ FAs), are monounsaturated lipids with a double-bond seven atoms away from the methyl end of the acyl chain, including palmitoleic acid $\left(16: 1 \Delta^{9}\right)$, and its elongation products, vaccenic acid (cis-18:1 $\left.\Delta^{11}\right)$ and paullinic acid $\left(\right.$ cis-20:1 $\left.\Delta^{13}\right) . \omega-7$ FAs are high-valued in human healthcare, but also in multiple industrial applications, ranging from the precursor of 1-octene for polyethylene to the feedstock of biodegradable lubricant and biodiesel (Curb et al., 2000; Misra et al., 2010; Mozaffarian et al., 2010; Nguyen et al., 2010, 2015). Unfortunately, such valued $\omega-7$ FAs are very low abundance in commercialized field oilseeds like cotton and soybean (Glycine max). Only a few of uncommercialized plants can synthesize and accumulate much high level of $\omega-7$ FAs in their seeds (Wu et al., 2012). For example, contents of $\omega-7$ FAs are high up to $64 \%, 30 \%$, and $32 \%$ in the mature seeds of cat's claw (Doxantha unguis-cati L.), macadamia (Macadamia integrifolia), and sea buckthorn (Hippophae rhamnoides), respectively (Cahoon et al., 1998; Gummeson et al., 2000; Kallio et al., 2002). Interestingly, $\omega-7$ FAs were found to be highly concentrated in the endosperm (aleurone) but not in the embryo in the seeds of Arabidopsis thaliana and rapeseed (Brassica napus), with $15 \mathrm{~mol} \%$ in Arabidopsis and $35 \%$ in rapeseed, respectively (Bryant et al., 2016). It is noteworthy that the endosperm is reduced to a single cell layer of residues surrounding the embryo in these mature seeds (Penfield et al., 2004; Li et al., 2006). Such a thin peripheral aleurone and seed coat are tightly stuck together, and consequently are stripped off the embryo during the dehulling process before oil extraction. The detailed molecular mechanism responsible for such high biosynthesis of $\omega-7$ FAs in the resource plants but not in the embryo of common oilseeds remains to be elucidated despite a number of enzymes and transcriptional factors were found to play important roles in formation of $\omega-7$ FAs (Bryant et al., 2016; Troncoso-Ponce et al., 2016).

In plants, fatty acyl-ACPs of different chain lengths and saturation levels are initially biosynthesized in plastid. Then, fatty acyl-ACPs are catalyzed by fatty acyl-ACP thioesterases to release free fatty acids chains, which are transported from plastid via cytosol into endoplasmic reticulum (ER) for functional modifications, such as acyl chain elongation and further desaturation. Finally, fatty acids are channeled into triacylglycerol (TAG) by cascade acylation reactions. Alternatively, fatty acids could also be integrated into phosphatidylcholine (PC) and other acyl carrier molecules.

It is believed that $\omega-7$ FAs are produced by $\Delta^{9}$ desaturation of palmitic acid $(16: 0)$ to form palmitoleic acid $\left(16: 1 \Delta^{9}\right)$, which can then be elongated (Cahoon et al., 1998). Such $\Delta^{9}$ desaturation is catalyzed by acyl-ACP $\Delta^{9}$ desaturase (AAD, EC 1.14.99.6) family (Parvini et al., 2016). The archetype for this family is the stearoylACP $\Delta^{9}$ desaturase (named shortly as SAD) which normally desaturates stearic acid (18:0) to form oleic acid $\left(18: 1 \Delta^{9}\right.$, cisw9) at high efficiency in plants. However, various AAD isoforms also exhibit different levels of substrate specificity on 16:0ACP (McKeon and Stumpf, 1982). Those AAD isoforms with preference on 16:0-ACP instead of 18:0-ACP as substrate are also named as palmitoyl-ACP $\Delta^{9}$ desaturases (PADs), and some of which were isolated from several high accumulators of $\omega-7$ FAs (Cahoon et al., 1997, 1998).

It is therefore recognized that the specific activity of SAD enzymes for substrates determines the ratio of $16: 1 \Delta^{9}$ to $18: 1 \Delta^{9}$ and their derivatives ( $\omega-7$ and $\omega-9$ FAs) in plant seeds. The key amino acids located in the catalytic domain were reported to play important roles for the substrate specificity of SAD enzymes (Whittle and Shanklin, 2001; Zhang Y. et al., 2015), but knowledge is still limited for the molecular determination of such substrate specificity. Recently, Arabidopsis AtAAD2 and AtAAD3 were identified as the major contributors for the synthesis of $\omega-7$ FAs in the aleurone, though seven AtAAD family members were detected in Arabidopsis genome (Bryant et al., 2016; Troncoso-Ponce et al., 2016).

As described above, cottonseeds contain high level of 16:0 $(\sim 25 \%)$ and $18: 2 \Delta^{9,12}(>50 \%)$, but little $16: 1 \Delta^{9}(<1 \%)$ and $18: 1 \Delta^{11}(<1 \%)$, showing that SAD enzymes responsible for $\Delta^{9}$ desaturation in upland cotton may have very higher selectivity for 18:0-ACP than for 16:0-ACP. However, RNAi down-regulation of KASII resulted in remarkable increase of $16: 1 \Delta^{9}$ and $18: 1 \Delta^{11}$ in cotton seeds by $10 \%$ and $1.5 \%$, respectively, indicating the existence of SAD isoforms having high specific activity to catalyze 16:0-ACP to 16:1-ACP (Liu et al., 2017). More importantly, we examined fatty acid profiles in separate tissues from cotton seeds, revealing that a certain amount of $16: 1 \Delta^{9}$ were concentrated in developing endosperm but not in embryo. Overall, there should be existed some SAD members responsible for biosynthesis of $16: 1 \Delta^{9}$ in upland cotton. How many members of SAD family are in allotetraploid upland cotton? Is there any SAD isoform having a higher substrate specificity toward 16:0-ACP or 18:0$\mathrm{ACP}$ ? If so, what are the distinct structure features between 16:0and 18:0-specific SAD isoforms? How they work coordinately in controlling the biosynthesis of $\omega-7$ and $\omega-9$ FAs in cotton seeds? Particularly, which members of SAD family in cotton are responsible for $\omega-7$ FA enrichment in developing endosperm but not in embryo? Since endosperm would be vanished from the mature seeds, what physiological roles are ascribed to $\omega-7$ FAs exclusively accumulated in developing endosperm? All these questions need to be addressed.

Here, a genome-wide characterization was performed to identify candidate SAD family members in upland cotton (Gossypium hirsutum L.), a naturally occurring allotetraploid $\left(2 \mathrm{n}=4 \mathrm{x}=(\mathrm{AD})_{2}=52\right)$ produced by interspecific hybridization 
of $\mathrm{A}$ - and D-genome diploid progenitor species (Zhang $\mathrm{T}$. et al., 2015). Analysis on the key amino acids in the functional domain of GhSAD members and their cis-elements as well as $3 \mathrm{D}$ structure were conducted to identify the putative $16: 0$ specific GhSADs. And then, the temporal-spatial expression patterns of these GhSAD genes were examined by both RNAseq data and qRT-PCR in various cotton tissues and developing seeds. Subsequently, for functional analysis of the target GhSADs, we employed the transient expression of these GhSAD genes in tobacco (Nicotiana benthamiana) leaf tissues and the heterologous expression in yeast (Saccharomyces cerevisiae) mutant BY4389 lacking the synthesis of unsaturated fatty acids, followed by detecting fatty acid profiles in the target samples. In the whole, the present data evidence that GhA-SAD6 and GhDSAD8 are responsible for making palmitoleic acid in developing cotton endosperm, providing novel insight to understand oil and $\omega-7$ FA biosynthesis in plant seeds and scientific reference for lipid metabolic engineering in commercial oilseeds.

\section{MATERIALS AND METHODS}

\section{Plant Materials}

Upland cotton (G. hirsutum cv. Zhong Mian 21) was planted in the experimental station at Shanxi Agricultural University, Taigu, China. Roots, stems and leaves were sampled from 6-weekold seedlings. Whole flower at 0 day after flowering (DAF) and developing ovules were sampled at 5, 10, 15, 20, 25, 35, and 45 DAF. Then ovules at 15, 25, 35 and 45 DAF were dissected manually to separate the embryos from the endosperms. All samples were instantly frozen in liquid nitrogen and stored at $-70^{\circ} \mathrm{C}$ for subsequent experiment. Tobacco ( $N$. benthamiana) was grown in an artificial incubator keeping at $26^{\circ} \mathrm{C}$ with $60 \%$ relative humidity and $14 \mathrm{~h}$ light/10 $\mathrm{h}$ dark photoperiod (Zhang et al., 2014).

\section{Gene Identification, Functional Domain, Three-Dimensional Model and Promoter Analysis}

The conserved FA_desaturase_2 domain (PF03405) was retrieved from Pfam database ${ }^{1}$ and used as the query to perform BLAST against proteins of $G$. hirsutum from the CottonGen database ${ }^{2}$. The sequence features were identified by HMMER version 3.0 with default parameters, and their conserved motifs were further verified by $\mathrm{SMART}^{3}$ and $\mathrm{CDD}$ tool $^{4}$. Only the sequence containing conserved FA_desaturase_2 domain was employed for further analysis. All candidate GhSAD genes were renamed according to their chromosomal distributions, denoting GhA$S A D 1 \sim G h A-S A D 9$ and $G h D-S A D 1 \sim G h D-S A D 8$. Transcriptional data were downloaded from cotton research institute of Nanjing Agricultural University ${ }^{5}$.

\footnotetext{
${ }^{1}$ http://pfam.xfam.org/

${ }^{2}$ https://www.cottongen.org/

${ }^{3} \mathrm{http}: / /$ smart.embl-heidelberg.de/

${ }^{4}$ http://www.ncbi.nlm.nih.gov/Structure/cdd/cdd.shtml

${ }^{5} \mathrm{http}: / /$ mascotton.njau.edu.cn/info/1054/1118.htm
}

For cis-element analysis, the extracted 2-kb upstream sequences from the initiation codon of all GhSADs were searched in the PlantCARE database ${ }^{6}$. The data of AtSAD proteins were downloaded from TAIR database ${ }^{7}$. Castor SAD sequences and $\mathrm{SAD}$ in cat's claw (Muc-PAD) were downloaded from $\mathrm{NCBI}^{8}$. To investigate the key amino acid residues that influence the catalytic properties of SAD enzyme, amino acid sequence comparisons of all GhSAD proteins and the reference protein sequences (RcSAD1, AtFAB2, AtAAD3, AtAAD2, and Muc-PAD) were conducted. Then, three-dimensional structures of GhSADs were modeled using Swiss-model ${ }^{9}$, with the crystal structure of RcSAD1 (NP_001310659.1) as the templet. In addition, the key amino acids and ligands in catalytic activity center were marked using Discovery Studio 4.1 software, with the default parameters.

\section{RNA Isolation and Real-Time Quantitative PCR}

EASYspin Plus Plant RNA Kit (Aidlab, Beijing, China) was used for the isolation of total RNA from all sample types. First-strand cDNA was reversely transcripted using PrimeScript ${ }^{\mathrm{TM}}$ II 1st Strand cDNA Synthesis Kit (Takara, Kyoto, Japan) according to the manufacturer's instruction. Gene-specific primers used were listed in Supplementary Table S1. The experiment was carried out on a Bio-Rad CFX96 Real-Time PCR system (BioRad, United States) with three replicates. Following reaction conditions were applied: $95^{\circ} \mathrm{C}$ for $10 \mathrm{~min}$; 40 cycles of $95^{\circ} \mathrm{C}$ for $15 \mathrm{~s}, 60^{\circ} \mathrm{C}$ for $1 \mathrm{~min} ; 72^{\circ} \mathrm{C}$ for $20 \mathrm{~s}$. The melting curve was analyzed after the final cycle, ramping from 65 to $95^{\circ} \mathrm{C}$ with increment $0.5^{\circ} \mathrm{C} / 5 \mathrm{~s}$. The normalized relative expression was calculated using the $2^{-\Delta \Delta \mathrm{Cq}}$ method (Livak and Schmittgen, 2001). Cotton EF-1 $\alpha$ (DQ174251) and histone 3 (AF024716.1) genes were employed as the internal references (Jiang et al., 2012; Shang et al., 2015).

\section{Construction of Plant and Yeast Expression Vectors of the GhSAD Members}

To test their substrate preferences, the full-length open reading frames (ORFs) of four representative GhSAD genes (GhA-SAD5, GhA-SAD6, GhA-SAD7, and GhD-SAD8) were amplified by PCR with oligonucleotides (Supplementary Table S2) and then cloned into pCAMBIA1303 vector under the drive of cauliflower mosaic virus (CaMV) $35 \mathrm{~S}$ promoter. The resulting vectors were transferred into Agrobacterium strain of GV3101 using the freeze-thaw method for further transient expression in tobacco leaves by infiltration. The empty vector pCAMBIA1303 was used as blank control. For functional analysis using yeast expression system, the target GhSAD genes with optimized codons were then cloned into the yeast expression vector pYES2 under the control of the GAL1 promoter. Additionally, AtFAB2 and AtAAD3 from $A$. thaliana were cloned according to the published sequences

\footnotetext{
${ }^{6}$ http://bioinformatics.psb.ugent.be/webtools/ plantcare/

${ }^{7}$ http://arabidopsis.org/

${ }^{8}$ http://www.ncbi.nlm.nih.gov/

${ }^{9}$ https://swissmodel.expasy.org/
} 
and inserted into pYES2 as positive controls. Empty vector of pYES2 was set as blank control. Related primers were listed in Supplementary Table S2.

\section{Transient Expression Assays in $N$. benthamiana Leaves}

The constructed plant expression vectors were transferred into Agrobacterium tumefaciens strain GV3101. Agroinfiltration was employed for transient expression of the target genes in tobacco leaves. Briefly, the bacteria bearing the vector were cultured in $\mathrm{LB}$ medium and incubated at $28^{\circ} \mathrm{C}$ overnight with $250 \mathrm{rpm}$. Bacterial cultures were then centrifuged at 8,000 rpm for $3 \mathrm{~min}$ and resuspended into fresh infiltration medium supplemented with acetosyringone, $\mathrm{MgSO}_{4}$ and MES to an optical density of 0.5 at $600 \mathrm{~nm}$. Agroinfiltration was performed using a needle-free syringe in the leaves of six-week-old $N$. benthamiana plants. The treated leaves were harvested 5 days later, and then ground up into powder for the extraction of total lipids.

\section{Yeast Culture and Transformation}

The mutant yeast S. cerevisiae strain BY4389 (MATa ole1 $\triangle$ ::LEU2 ura3-52 his4) that cannot synthesize unsaturated fatty acids was purchased from Osaka University, Japan (Xue et al., 2016). Before transformation, the cells were cultivated at $28^{\circ} \mathrm{C}$ in modified yeast-extract peptone dextrose (YPD) medium (0.01\% sodium oleate, $1 \%$ yeast extract, $2 \%$ peptone and $2 \%$ glucose) with shaking $(200 \mathrm{rpm})$. When the concentration was at OD600 $\approx 0.5$, the cells were harvested by centrifugation, washed three times with precooling sterilized water. Then, the cells were resuspended in $100 \mathrm{mM}$ LiAc solution. After removing LiAc, we got the competent cells of yeast with high transformation efficiency. The constructed yeast expression vector and empty pYES2 were separately transformed into S. cerevisiae mutant strain BY4389 using Yeast Transformation Kit (Coolaber, Beijing, China). Transformed cells were selected on the modified YPD medium lacking uracil (SC-Ura). After cultured 3 days at $28^{\circ} \mathrm{C}, \mathrm{PCR}$ confirmation was used to select positive clones. The correct transformed cells were then transferred in the corresponding selective medium to increase the yeast biomass for $24 \mathrm{~h}$. For induction, glucose was replaced by galactose $(2 \%, \mathrm{w} / \mathrm{v})$ and sodium oleate was removed, and cells were further cultivated for another $72 \mathrm{~h}$. At last, transformed cells were harvested and freeze-dried into powder to for fatty acid analysis.

\section{Lipid Extraction and Fatty Acid Methyl Ester Analysis}

Total lipids from developing cotton embryo and endosperm (15, 25, 35 and $45 \mathrm{DAF}$ ), the wild and transgenic N. benthamiana leaves and yeast samples were extracted, and then converted into fatty acid methyl esters (FAMEs) as previously described (Bhattacharya et al., 2015). Briefly, FAMEs were transmethylated using $2 \mathrm{~mL}$ of methanol that contained 2.5\% sulfuric acid (v/v) and then incubated at $85^{\circ} \mathrm{C}$ for $2 \mathrm{~h}$. For quantification of the FAs, an appropriate amount of C17:0 FAME (Sigma) was added as internal standard. FAMEs were analyzed by Agilent gas chromatograph equipped with a flame ionization detector and a capillary column (HP-88 $100 \mathrm{~m} \times 0.25 \mathrm{~mm} \times 0.2 \mu \mathrm{m})$. Nitrogen was used as carrier gas with a flow rate of $2.64 \mathrm{ml} / \mathrm{min}$. $1 \mu \mathrm{L}$ of the sample was injected into the column with three replicates. The injection and detector temperatures were both set as $250^{\circ} \mathrm{C}$. The gas chromatograph oven was programmed at an initial temperature of $140^{\circ} \mathrm{C}$ for $5 \mathrm{~min}$, and then increased up to $250^{\circ} \mathrm{C}$ at a rate of $15^{\circ} \mathrm{C} / \mathrm{min}$. Fatty acid component was estimated according to the retention time of fatty acid standard and data was collected by peak area normalization with C17:0 internal standard.

\section{RESULTS}

\section{Differential Distribution of Fatty Acids Between Embryos and Endosperms in Developing Cottonseeds, Especially for Palmitoleic Acid}

Angiosperm seed formation is characterized by the double fertilization of the embryo sac. During this process, one sperm cell fertilizes the egg cell to produce a zygote, which develops into an embryo, whereas the second sperm cell targets the central cell polar nuclei, leading to the endosperm formation. Both the diploid embryo and the triploid endosperm are enclosed by the maternally derived ovular integument layers that form seed coat. As a typical exalbuminous seed, cotton seed has no endosperm left at mature stage despite abundant endosperms surrounding the embryo are generated during the early seed development, providing nutrition for the enlarging embryo as it develops (Figure 1A).

To investigate whether fatty acid profiles are different between embryos and endosperms during development, we harvested embryo and endosperm samples, respectively, at four stages of seed development denoted as 15 DAF (days after flowering), 25, 35, and 45 DAF (Figure 1A). At the early stage of 15 DAF, the endosperm forms a thick milky layer surrounding the embryo. During seed development and maturation, the endosperm volume is gradually reduced into a thin layer closely to seed shell (25-35 DAF), accompanied by the embryo growing. At the end of seed development (45 DAF), the endosperm is disappeared in cotton seed.

The examination of FA contents in developing cotton seeds (Figure 1B) showed that FA profiles were significant different between endosperm and embryo. The most difference is that $16: 1 \Delta^{9}$, a kind of $\omega-7$ FAs, was largely accumulated in endosperm with the peak at $25 \mathrm{DAF}$ (16.95 Mol\% of total FAs), but only trace level $(<1 \mathrm{Mol} \%)$ in embryo. However, content of $18: 1 \Delta^{9}$, a kind of $\omega-9$ FAs, was higher in embryo than in endosperm by 1.2-1.7 folds, with the maximum level (15.04 Mol\%) in embryo at 35 DAF. Palmitic acid (16:0) was the major FA in endosperm at $15 \mathrm{DAF}$, and it reduced gradually during seed development, with lower level in endosperm than in embryo from 25 to $35 \mathrm{DAF}$. Linoleic acid $\left(18: 2 \Delta^{9,12}\right)$, the major FA in embryo, is increased up to $\sim 60 \%$ in embryo at $25 \mathrm{DAF}$, with lower level in developing endosperm. The contents of 18:0 and 18:3 $\Delta^{9,12,15}$ were not significant between embryo and endosperm from 25 to 35 DAF 
A
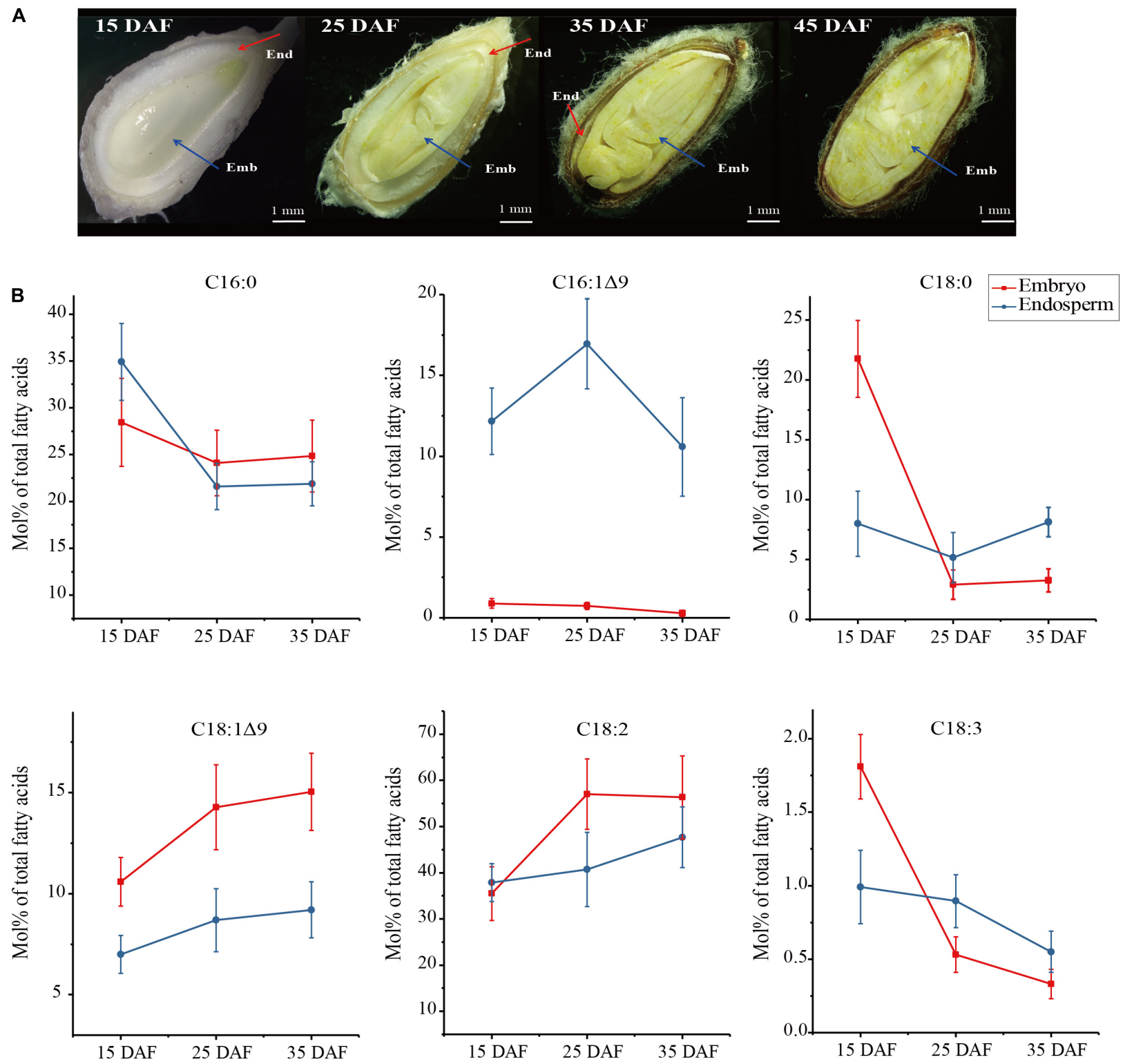

FIGURE 1 | Four development stages of cotton seeds (A) and fatty acid profiles in developing embryo and endosperm (B). Fatty acid content (Mol\%) was presented as a percentage of all fatty acids in embryo and endosperm, respectively. Values are the mean \pm SE of six biological duplicates.

despite these two fatty acid contents were higher in embryo than in endosperm at 15 DAF of the seed development. Notably, abundant palmitoleic acid $\left(16: 1 \Delta^{9}\right)$ were found to be selectively accumulated in developing endosperm, suggesting that some unknown mechanism may control this differential accumulation of palmitoleic acid between the endosperm and embryo.

\section{Identification of Seventeen Stearoyl-ACP $\Delta^{9}$ Desaturases (GhSADs) and Six Putative 16:0-ACP Specific GhSADs From Upland Cotton}

As described in the introduction, some members (if not all) of SAD family can catalyze palmitic acid (16:0)-ACP to form palmitoleic acid $\left(16: 1 \Delta^{9}\right)$-ACP in plant seeds. The preferred accumulation of $16: 1 \Delta^{9}$ in the developing cotton endosperm observed above indicates that SAD enzyme working in endosperm may have high substrate specificity for palmitic acid (16:0). To investigate such SAD members with high 16:0-ACP specificity, we performed a genomewide characterization of SAD family members in upland cotton (G. hirsutum) genome. Total of 17 putative GhSADs were identified according to the conserved domain and their integrity. These predicted GhSADs were designated as GhA$S A D 1 \sim G h A-S A D 9$ and GhD-SAD1 GhD-SAD8 based on chromosome number of A- or D-genome where they located (Supplementary Table S3). 
The known reports have established that RcSAD1 from castor (Ricinus communis) (Lindqvist et al., 1996) and AtFAB2 from Arabidopsis (Lightner et al., 1994) are characterized as the 18:0-ACP specific SADs while AtAAD2 and AtAAD3 from Arabidopsis (Troncoso-Ponce et al., 2016) as well as Muc-PAD from cat's claw (Macfadyena unguis-cati) (Cahoon et al., 1998) are identified as the 16:0-ACP specific enzymes. Moreover, the substrate specificity of these enzymes is determined by a group of eight amino acid residues comprising the region of the catalytic sites (also referred as the lower portion of the hydrophobic binding pocket for FA). To identify which GhSAD may be the candidate of 16:0-ACP specific enzyme, these well-characterized SADs were used as the references to perform in silico analyses of 17 GhSAD sequences including sequence comparisons and modeling of 3D protein structures (Arnold et al., 2006; Bordoli et al., 2009).

Table 1 summarized the comparison of the eight amino acid (AA) residues lining the lower portion of the hydrophobic binding pocket for FA in those enzymes tested. Seventeen GhSADs were clearly divided into two groups. Group 1 with ten GhSAD members had the identical AA residues in the conserved functional domains as RcSAD1 and AtFAB2 (Table 1), which are $\omega-9$ producing SAD isoforms. Group 2 consisting of GhA-SAD5, 6, 7, and GhD-SAD4, 5, 7, 8 were gathered together with 16:0-ACP specific Muc-PAD, AtAAD2 and AtAAD3, although divergent residues existed in the eightAA domain of those enzymes. The 3D model revealed that the eight $\mathrm{AA}$ residues were located near the bottom of the substrate-binding channel close to diiron center of the enzymes examined (Figure 2I). The identical eight-AA domain in the 18:0-ACP specific enzymes is predicted to shape a deep/long substrate cavity (pocket/channel) to accommodate 18:0-ACP substrate (Figure 2A). However, the divergent eight-AA domain in the 16:0-ACP specific enzymes is predicted to make the substrate channel shorter, which is more adaptable for 16:0-ACP substrate (Figures 2B,C,D).

To further test whether these GhSADs in Group 2 are the candidate enzymes responsible for $16: 1 \Delta^{9}$ biosynthesis in developing cotton endosperm, cis-elements analyses were conducted on the promoter sequences of the corresponding genes. The promoters of GhA-SAD5, GhA-SAD6, GhA-SAD7,

TABLE 1 | Comparison of the eight amino acid residues comprising the region of the catalytic sites of GhSADs.

\begin{tabular}{|c|c|c|c|c|c|c|c|c|c|c|c|}
\hline \multirow{2}{*}{$\begin{array}{l}\text { Ricinus communis } \\
\text { RcSAD1 }\end{array}$} & \multirow{2}{*}{$\begin{array}{c}\begin{array}{c}\text { Arabidopsis } \\
\text { thaliana }\end{array} \\
\text { AtFAB2 }\end{array}$} & \multicolumn{10}{|c|}{ Gossypium hirsutum } \\
\hline & & $\begin{array}{l}\text { GhA- } \\
\text { SAD1 }\end{array}$ & $\begin{array}{l}\text { GhA- } \\
\text { SAD2 }\end{array}$ & $\begin{array}{l}\text { GhA- } \\
\text { SAD3 }\end{array}$ & $\begin{array}{l}\text { GhA- } \\
\text { SAD4 }\end{array}$ & $\begin{array}{l}\text { GhA- } \\
\text { SAD8 }\end{array}$ & $\begin{array}{l}\text { GhA- } \\
\text { SAD9 }\end{array}$ & $\begin{array}{l}\text { GhD- } \\
\text { SAD1 }\end{array}$ & $\begin{array}{l}\text { GhD- } \\
\text { SAD2 }\end{array}$ & $\begin{array}{l}\text { GhD- } \\
\text { SAD3 }\end{array}$ & $\begin{array}{l}\text { GhD- } \\
\text { SAD6 }\end{array}$ \\
\hline \multicolumn{12}{|c|}{ Group 1: $\omega-9$ producing isoforms } \\
\hline L118 & L156 & $\mathrm{L} 141$ & L150 & L140 & L151 & L150 & L150 & L150 & L141 & L151 & L150 \\
\hline F189 & F227 & F212 & F211 & F211 & F212 & F221 & F221 & F221 & F212 & F222 & F221 \\
\hline L115 & L153 & 1138 & L147 & 1137 & L148 & $\mathrm{L} 147$ & $\mathrm{~L} 147$ & L147 & 1138 & L149 & $\mathrm{L} 147$ \\
\hline $\mathrm{T} 117$ & T155 & $\mathrm{T} 140$ & T149 & T139 & $\mathrm{T} 150$ & $\mathrm{~T} 149$ & $\mathrm{~T} 149$ & T149 & $\mathrm{T} 140$ & T150 & $\mathrm{T} 149$ \\
\hline G188 & G226 & G211 & G210 & G210 & G211 & G220 & G220 & G220 & G211 & G221 & G220 \\
\hline $\mathrm{T} 181$ & T219 & T204 & $\mathrm{T} 213$ & T203 & $\mathrm{T} 214$ & $\mathrm{~T} 213$ & $\mathrm{~T} 213$ & $\mathrm{~T} 213$ & T204 & T214 & $\mathrm{T} 213$ \\
\hline P179 & P217 & P202 & P211 & P201 & P212 & P211 & $\mathrm{P} 211$ & P211 & P202 & P212 & P211 \\
\hline M114 & M152 & M137 & M146 & M136 & M147 & M146 & M146 & M146 & M137 & M148 & M146 \\
\hline \multicolumn{2}{|c|}{ Arabidopsis thaliana } & \multicolumn{2}{|c|}{ Dauricum unguis-cati } & \multicolumn{7}{|c|}{ Gossypium hirsutum } & \\
\hline AtAAD3 & AtAAD2 & & Muc-PAD & $\begin{array}{l}\text { GhD- } \\
\text { SAD4 }\end{array}$ & $\begin{array}{l}\text { GhA- } \\
\text { SAD5 }\end{array}$ & $\begin{array}{l}\text { GhD- } \\
\text { SAD5 }\end{array}$ & $\begin{array}{l}\text { GhA- } \\
\text { SAD6 }\end{array}$ & $\begin{array}{l}\text { GhA- } \\
\text { SAD7 }\end{array}$ & $\begin{array}{l}\text { GhD- } \\
\text { SAD7 }\end{array}$ & $\begin{array}{l}\text { GhD- } \\
\text { SAD8 }\end{array}$ & \\
\hline \multicolumn{4}{|c|}{$\omega-7$ producing isoforms } & \multicolumn{7}{|c|}{ Group 2: $\omega-7$ producing isoforms? } & \\
\hline L153 & L163 & & W152 & $\mathrm{L} 151$ & L31 & L150 & $\mathrm{L} 152$ & M148 & M148 & L151 & \\
\hline F224 & F234 & & F223 & F222 & F102 & F221 & F223 & A219 & A219 & Y222 & \\
\hline L150 & L160 & & L149 & L149 & L28 & $\mathrm{L} 147$ & L149 & $\mathrm{L} 145$ & L145 & L148 & \\
\hline $\mathrm{T} 152$ & T162 & & T151 & $\mathrm{T} 150$ & $\mathrm{R} 30$ & R149 & $\mathrm{T} 151$ & $\mathrm{~T} 147$ & $\mathrm{~T} 147$ & T150 & \\
\hline G223 & G233 & & G222 & G221 & $\mathrm{T} 101$ & T220 & T222 & G218 & G218 & A221 & \\
\hline F216 & F226 & & T215 & T214 & 194 & 1213 & A215 & $\mathrm{S} 211$ & S211 & A214 & \\
\hline S214 & S224 & & P213 & P212 & V192 & V211 & P213 & S209 & S209 & M212 & \\
\hline T149 & T159 & & M148 & 1148 & 127 & M146 & M148 & L144 & L144 & M147 & \\
\hline
\end{tabular}

Sequences were conducted by GeneDoc software using RcSAD1, AtFAB2, AtAAD3 as well as Muc-PAD as temples. The GenBank or TAIR accession numbers of the

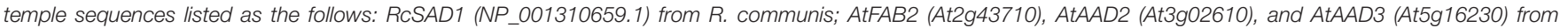

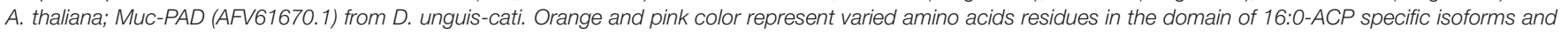
the putative 16:0-ACP specific GhSADs, respectively, compared to the 18:0-ACP specific isoforms characterized. 


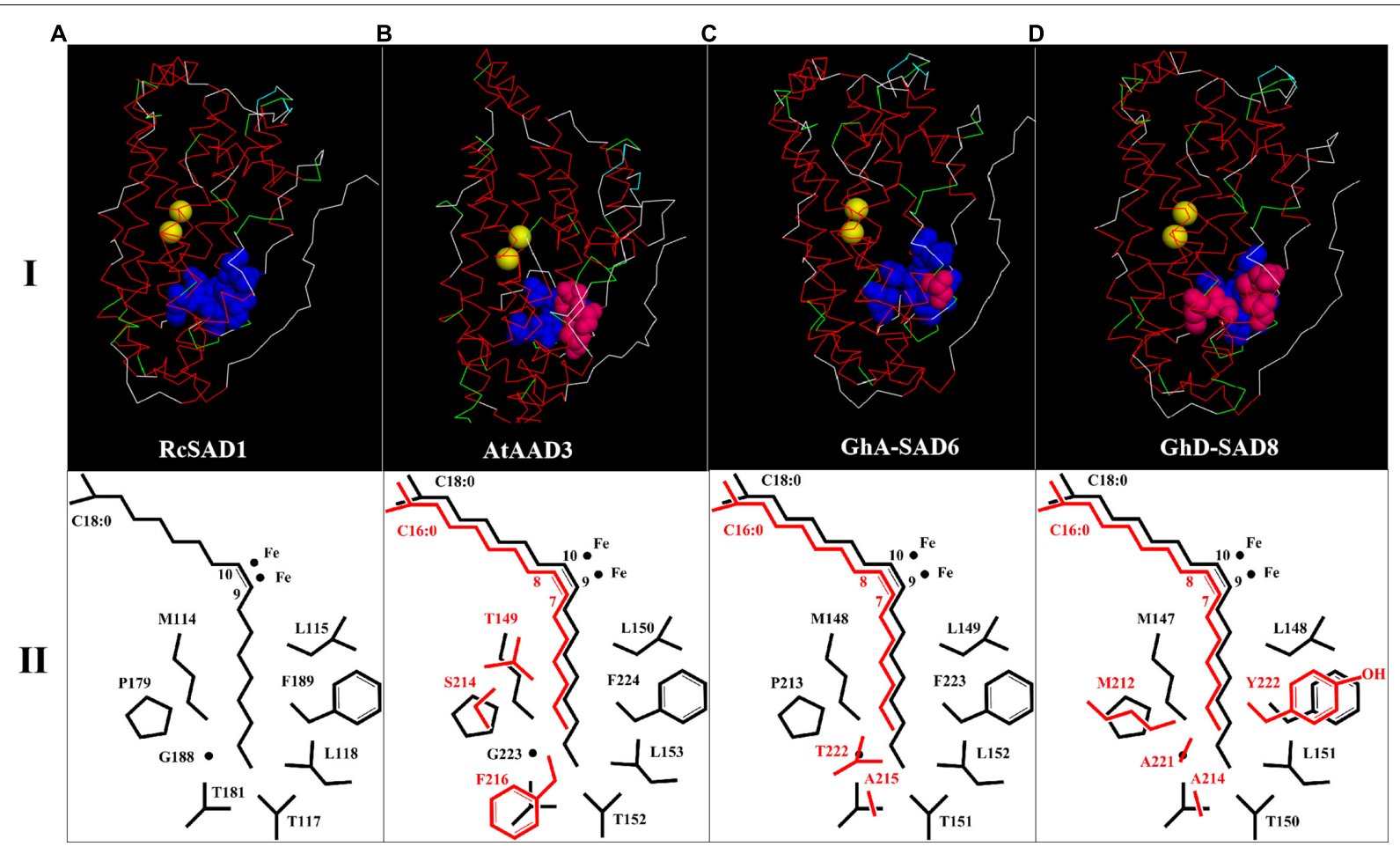

FIGURE 2 | 3D structure models of GhSAD protein monomers (C,D, upper panel I) and C18:0/C16:0-ACP chains as well as side chains of key amino acids close to catalytic center of diiron ions (lower panel II). All 3D models were predicted on Swiss-model (https://swissmodel.expasy.org/) with RcSAD1 (NP_001310659.1) and AtAAD3 as temples (A,B). Files of 3D structure of GhSADs were showed using Discovery Studio 4.1 software. Yellow bolls in upper panel I refer to diiron ions. Blue bolls/spheres refer to common amino acids as same as RcSAD1 while pink bolls refer to the varied amino acids. The fatty-acyl chains and side chains of amino acids were drawn by ChemDraw software (lower panel II). Black color represents C18:0-ACP and its common amino acids. Red indicates C16:0-ACP and varied amino acids.

GhD-SAD5, GhD-SAD7 and GhD-SAD8 contained multiple skn1 motifs with the core sequences of GTCAT which is necessary for gene expression in endosperm (Zhang et al., 2013). In addition, GhD-SAD5 and GhD-SAD8 have another GCN4 motif (the core sequences is TGAGTCA) involved in gene expression in endosperm (Supplementary Table S4) (Song et al., 2012). However, the promoter of GhD-SAD4 lacked such cis-element. Overall, sequence analysis indicate that GhA-SAD5, 6, 7, and GhD-SAD5, 7, 8 may be the putative 16:0-ACP specific SADs responsible for palmitoleic acid biosynthesis in developing cotton endosperm.

\section{Expression Patterns of GhSAD Genes in Different Cotton Tissues}

If any of GhSADs identified above functions in endosperm palmitoleic acid production, it must be expressed in developing cotton endosperm. To determine this, we firstly examined their transcript abundance during developing ovules using cotton RNA-seq profiles publically available (see text footnote 5). As shown in Figure 3, these 17 GhSADs exhibited different expression patterns in developing ovules. High expressions of GhD-SAD7, GhA-SAD3, and GhD-SAD4, occurred at the early stages of ovules. GhA-SAD2, GhD-SAD1 and GhD-SAD3 abundantly appeared at 5 DAF. Seven GhSAD (GhA-SAD5,
GhA-SAD6, GhD-SAD5, GhA-SAD-7, GhD-SAD8, GhA-SAD1, and $G h D-S A D 2)$ transcripts greatly accumulated at the middle stage of ovules (from $10 \mathrm{DAF}$ to $25 \mathrm{DAF}$ ), while $G h A$ SAD4 and GhD-SAD6 largely expressed at the late stage of ovules (35 DAF).

To further verify the expression patterns of these GhSADs, we employed qRT-PCR to investigate their mRNA levels in various cotton tissues (Figure 4). Six GhSADs with high expressions were selected for this verification. Specific primers for qRT-PCR were designed at 3/ untranslated regions of the GhSADs because of their highly conserved ORF sequences (Supplementary Table S1). As shown in Figure 4, GhA-SAD5, GhA-SAD6, and $G h D-S A D 8$ were highly expressed in the early middle developing ovules (5 and $10 \mathrm{DAF}$ ) while $G h A-S A D 7$ enriched at the late developing stage (20 DAF). GhA-SAD7 also had the highest expression level in root compared to other tissues, indicating that GhA-SAD7 may function importantly in root. For flower tissue, both GhD-SAD5 and GhD-SAD7 had abundant expression levels, implying that GhD-SAD5 and GhD-SAD7 play roles in flower development. It is known that the fastest period of oil/FA biosynthesis in cotton endosperm was the middle stage (1015 DAF) of the developing ovule. Combination of RNA-seq and qRT-PCR data suggests that GhA-SAD6, GhD-SAD8, GhASAD5, and GhA-SAD7 may play roles in FA/oil biosynthesis in developing cotton endosperm. 


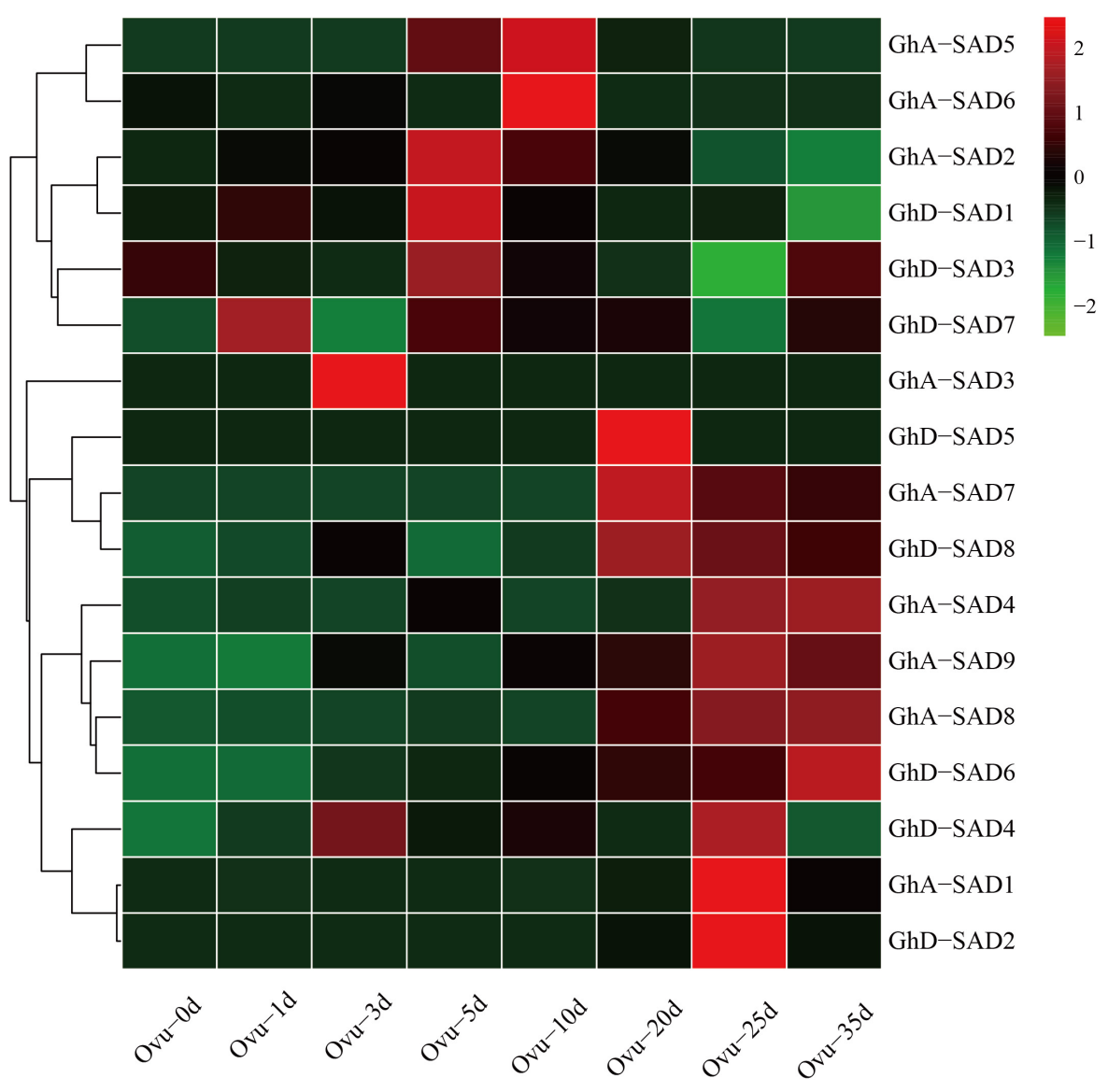

FIGURE 3 | Transcriptional expression patterns of 16 GhSAD family members during developing cotton ovule. The RNA-seq data used here were derived from NCBI database. The developing ovule stages were listed as 0d, 1d, 3d, 5d, 10d, 20d, 25d, and 35d after flowering (DAF) in developing cotton ovules.

\section{GhA-SAD6 and GhA-SAD8 Mostly Expressed in Developing Endosperm, Positively Correlating With Palmitoleic Acid Accumulation in Cotton Seeds}

To explore whether those GhSADs observed above are associated with $16: 1 \Delta^{9}$ production in endosperm, developing cotton seeds at $15,25,35$, and 45 DAF were then dissected and the two fractions, embryo and endosperm, were separately obtained to examine FA profiles and the expression patterns of the four GhSAD genes (GhA-SAD5, GhA-SAD6, GhA-SAD7, and GhD$S A D 8)$ were selected according to the above characterization.

As shown in Figure 5, the four GhSAD genes exhibited differential expression patterns between embryo and endosperm of cotton seeds. The mRNA levels of GhA-SAD5, GhA-SAD6, and $G h D-S A D 8$ were significantly high in the endosperm but very low in the embryo during seed development from 15 to 25 DAF, a period of palmitoleic acid level increased rapidly in the endosperm (Figures 5A,B,D). However, GhA-SAD7 expression was no obvious difference between embryo and endosperm (Figure 5C). Only a trace level of the four gene transcripts was detected in the embryo at the stage of 45 DAF. By this time, no endosperm was left in the seed. In addition, all these four genes had extremely low expression levels in cotton embryos and endosperms at 35 DAF compared to the two early stages (15 and 25 DAF). All these data suggest that GhA-SAD6 and GhD-SAD8 may be the main players for fast oil and palmitoleic acid accumulation in developing cotton endosperm although GhA-SAD5 has a higher expression in endosperm at $15 \mathrm{DAF}$.

To further confirm the association between GhA-SAD6 and $G h D-S A D 8$ expressions and palmitoleic acid level in the endosperm of developing cotton seeds, two set of data, MUFA (16:1 $\Delta^{9}$ and 18:1 $\left.\Delta^{9}\right)$ contents and expression levels of $G h A-S A D 6$ and $G h D-S A D 8$ were integrated into one figure (Figure 6). In the embryo, neither $16: 1 \Delta^{9}$ nor $18: 1 \Delta^{9}$ content change was obviously correlated with the expression level of either $G h A-S A D 6$ or $G h D-S A D 8$ gene (Figures $\mathbf{6 A}, \mathbf{C}$ ). In the endosperm, no correlation was also detected between 18:1 $\Delta^{9}$ content and the two gene expressions (Figure 6D). However, a significantly positive correlation was detected between 16:1 $\Delta^{9}$ content and GhA-SAD6 or GhD-SAD8 expression in the endosperm fractions (Figure 6B). Moreover, linear regression analysis also showed that the level of palmitoleic acid in the endosperm is positively correlated with the expression of $G h A$ SAD6 $(r=0.752)$ and GhD-SAD8 $(r=0.886)$, respectively, again indicating that GhA-SAD6 and GhD-SAD8 may be the key 


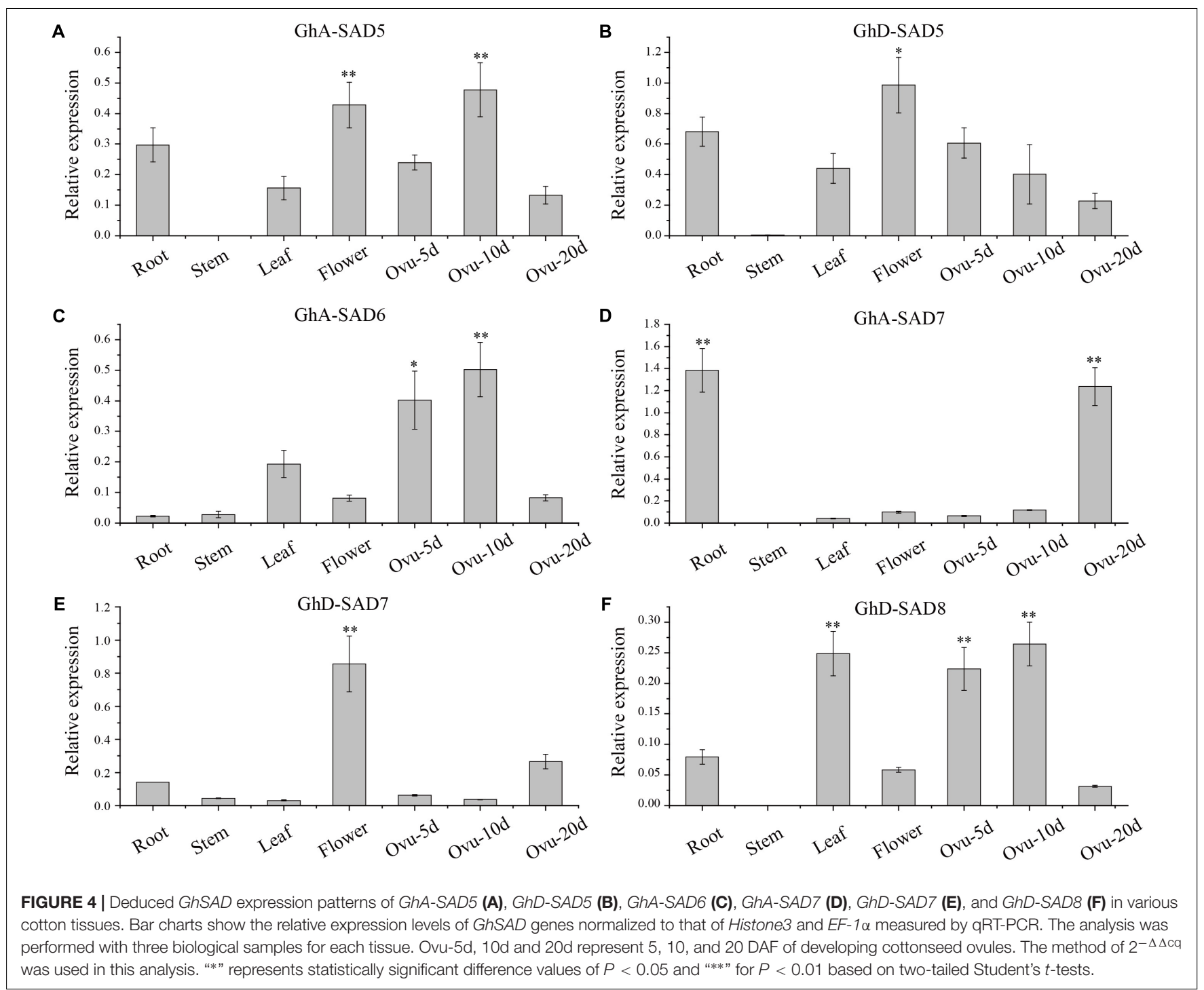

contributors for biosynthesis and accumulation of palmitoleic acid in cotton endosperm.

\section{GhA-SAD6 and GhD-SAD8 Boosted Accumulation of Palmitoleic Acid When Transient Expression in \\ $N$. benthamiana Leaves}

In order to identify the roles of GhA-SAD6 and GhD-SAD8 for biosynthesis of palmitoleic acid $\left(16: 1 \Delta^{9}\right)$, we transiently overexpressed them separately in leaves of $N$. benthamiana by Agrobacterium-mediated infiltrating. GhA-SAD5 and GhA-SAD7 were also transiently expressed, respectively, in tobacco leaves for comparison. The transformed leaves were harvested at 5 days after infiltration, and used for lipid extraction. FA analysis by GC (Figure 7) revealed that compared to the wild-type and emptyvector controls, either GhA-SAD6 or GhD-SAD8 overexpression led to a significant increase of palmitoleic acid by at least 45 folds. However, GhA-SAD5 or GhA-SAD7 expression alone resulted in a slight enhance of oleic acid $\left(18: 1 \Delta^{9}\right)$ by 2 folds around, with no effects on palmitoleic acid biosynthesis. These results indicate that GhA-SAD6 and GhD-SAD8 are specific for the ectopic enrichment of palmitoleic acid (e.g., to catalyze 16:0ACP into $16: 1 \Delta^{9}$-ACP) in the leaves whereas GhA-SAD5 and GhA-SAD7 are specific for oleic acid biosynthesis (e.g., to catalyze 18:0-ACP into 18:1 $\Delta^{9}$-ACP). Other changes of FA profiles were the increase of linoleic acid $\left(18: 2 \Delta^{9,12}\right)$ but reduction of linolenic acid $\left(18: 3 \Delta^{9,12,15}\right)$ in the GhA-SAD5- or GhA-SAD7-expressed leaves. Such change in polyunsaturated fatty acid levels maybe due to the activity changes of the endogenous NbFAD2 and NbFAD3 enzymes in tobacco leaves.

\section{GhA-SAD6 and GhD-SAD8 Accounted for Palmitoleic Acid Production When Expressed in Yeast Mutant BY4389}

To further confirm the function of GhA-SAD6 and GhD-SAD8 for biosynthesis of palmitoleic acid $\left(16: 1 \Delta^{9}\right)$, we overexpressed 


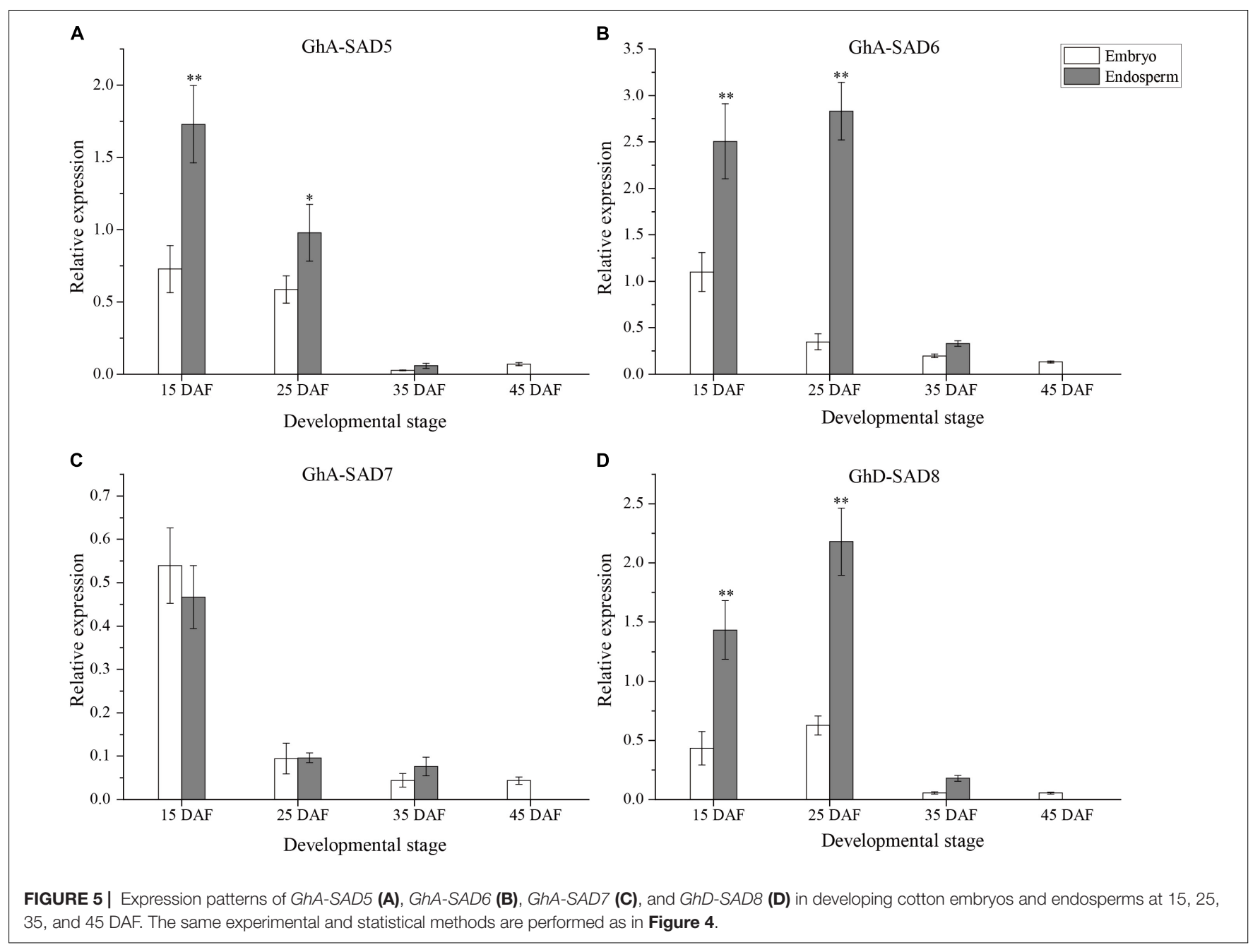

the four GhSAD genes (GhA-SAD5/6/7 and GhD-SAD8), respectively, in yeast mutant $B Y 4389$ disable in synthesis of unsaturated fatty acids. For this genetic complementary assays, Arabidopsis AtFAB2 (18:0-ACP specific) and AtAAD3 (16:0-ACP specific) were employed as the positive controls, and an empty pYES2 vector was used as the negative control. FA composition was examined in the transformed BY4389 strains.

Notably, all transformed yeast mutants except for empty pYES2 vector restored their desaturase function, and thus survived in SC-Ura medium without any unsaturated fatty acid. FA profiles detected (Figure 8) showed that the transgenic yeasts synthesized new but unbalanced-level unsaturated fatty acids (UFAs). As expected, AtAAD3-transgenic yeast generated $16.9 \mathrm{Mol} \%$ of $\mathrm{C} 16: 1 \Delta^{9}$ while AtFAB2-transgenics produced $19.03 \mathrm{Mol} \%$ of $\mathrm{C} 18: 1 \Delta^{9}$. Like in the AtAAD3transgenics, both GhA-SAD6- and GhD-SAD8-expressed yeast accumulated high levels of $\mathrm{C} 16: 1 \Delta^{9}$ by 13.83 and 9.88 Mol\%, respectively, but almost no detectable of $\mathrm{C} 18: 1 \Delta^{9}$. In contrast, the yeast mutants harboring GhA-SAD5 or GhA$S A D 7$ produced large amount of $C 18: 1 \Delta^{9}$ by approximately $10 \mathrm{Mol} \%$, with $<3.5 \mathrm{Mol} \%$ of $\mathrm{C} 16: 1 \Delta^{9}$, which was similar to FA profiles in the AtFAB2-transgenic yeast. Overall, the present data obtained by in vivo assays using yeast mutant transformation again revealed that GhA-SAD6 and GhD-SAD8 both had palmitoyl-ACP substrate specificity, with the former stronger. Whereas, GhA-SAD5 and GhA-SAD7 showed to prefer stearoyl-ACP substrate over palmitoyl-ACP despite of low enzymatic activities.

\section{DISCUSSION}

\section{Enrichment of $\omega-7$ FAs in Developing Cotton Seeds May Benefit the Embryo Development}

For the albuminous seeds such as cereal grains and castor beans, the embryo is embedded in the endosperm which persists to the mature seed stage as a storage tissue supplying nutrients for the seedling that will grow from the embryo (Huh et al., 2007). However, for exalbuminous seeds such as soybean, peanut and cotton, the endosperm is absorbed by the embryo during seed development, and the cotyledons 

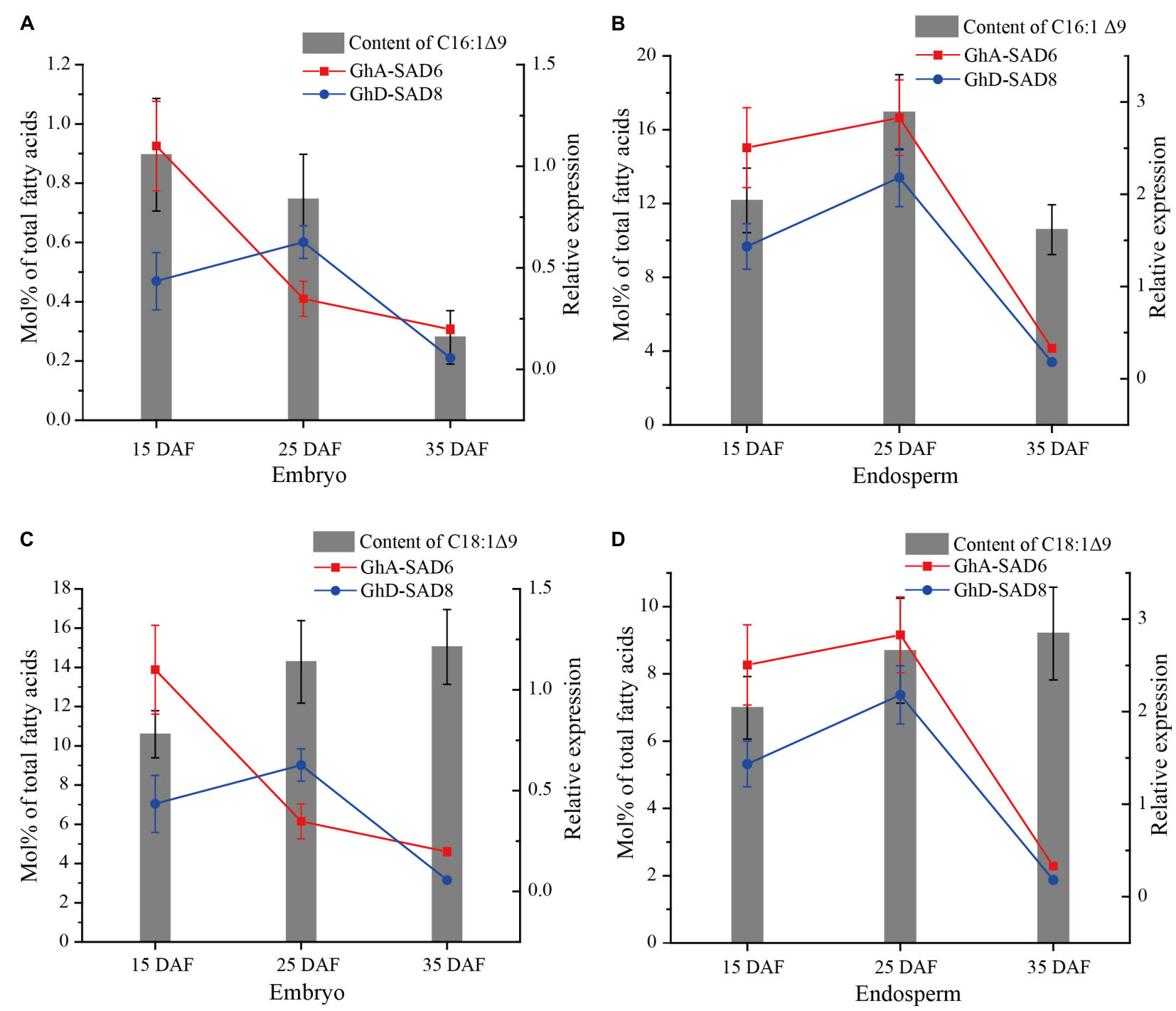

FIGURE 6 | The correlation of the expression of GhA-SAD6 and GhD-SAD8 with molar percentage of $\mathrm{C} 16: 1 \Delta^{9}$ or $\mathrm{C} 18: 1 \Delta^{9}$ in developing cotton embryos and endosperms. Columns showed fatty acid content (Mol\% of total fatty acids) and lines for relative expression (Red for GhA-SAD6, blue for GhD-SAD8). (A,C) represented the correlation between the fatty acid content $\left(C 16: 1 \Delta^{9}\right.$ or $\left.C 18: 1 \Delta^{9}\right)$ and relative expression levels of GhA-SAD6 or GhD-SAD8 in developing embryos, respectively. (B,D) were the correlation of $\mathrm{C} 16: 1 \Delta^{9}$ or $\mathrm{C} 18: 1 \Delta^{9}$ content with GhA-SAD6 or GhD-SAD8 in developing endosperms, respectively. Six biological replicates were analyzed in each tissue.

become filled with stored food (Yan et al., 2014). And thus, no endosperm is left in maturity seeds. A differential partitioning of reserve compounds between different tissues in Arabidopsis seeds was examined, with a dramatically increased accumulation of the economically important $\omega-7$ FAs in the endosperm, but not in the embryo (Penfield et al., 2004; Barthole et al., 2014). Here, we demonstrate that palmitoleic acid $\left(16: 1 \Delta^{9}\right)$ is enriched in developing cotton endosperm but not in the embryo. This $\omega-7$ FA accumulation is different in Arabidopsis and rapeseed where this unusual fatty acid is highly concentrated in a single cell layer (aleurone) of the residual endosperm surrounding the embryo in the mature seed, with the major species being cis-vaccenic acid $\left(18: 1 \Delta^{11}\right)$ and paullinic acid $\left(20: 1 \Delta^{13}\right)$ instead of palmitoleic acid $\left(16: 1 \Delta^{9}\right)$ (Li et al., 2006).
Such difference suggests that there may be different temporalspatial regulation mechanisms in different plant seeds. And it also raises the question of what is the biological function of these $\omega-7$ FAs enriched in the developing endosperm or the residual endosperm.

In Arabidopsis, transcriptional factors MYB115 and MYB118 coordinately upregulate the transcription of AtAAD2 and AtAAD3 which catalyze the synthesis of $\omega-7$ FAs, leading to high levels of $\omega-7$ FA accumulation exclusively in the endosperm of early maturing seeds (Bryant et al., 2016; Troncoso-Ponce et al., 2016). Arabidopsis double mutant of MYB115 and MYB118 resulted in significant decrease of $\omega-7$ FAs in the endosperm, concurrently with a slight delay in the elongation and enlargement of the embryo associated with a moderate 


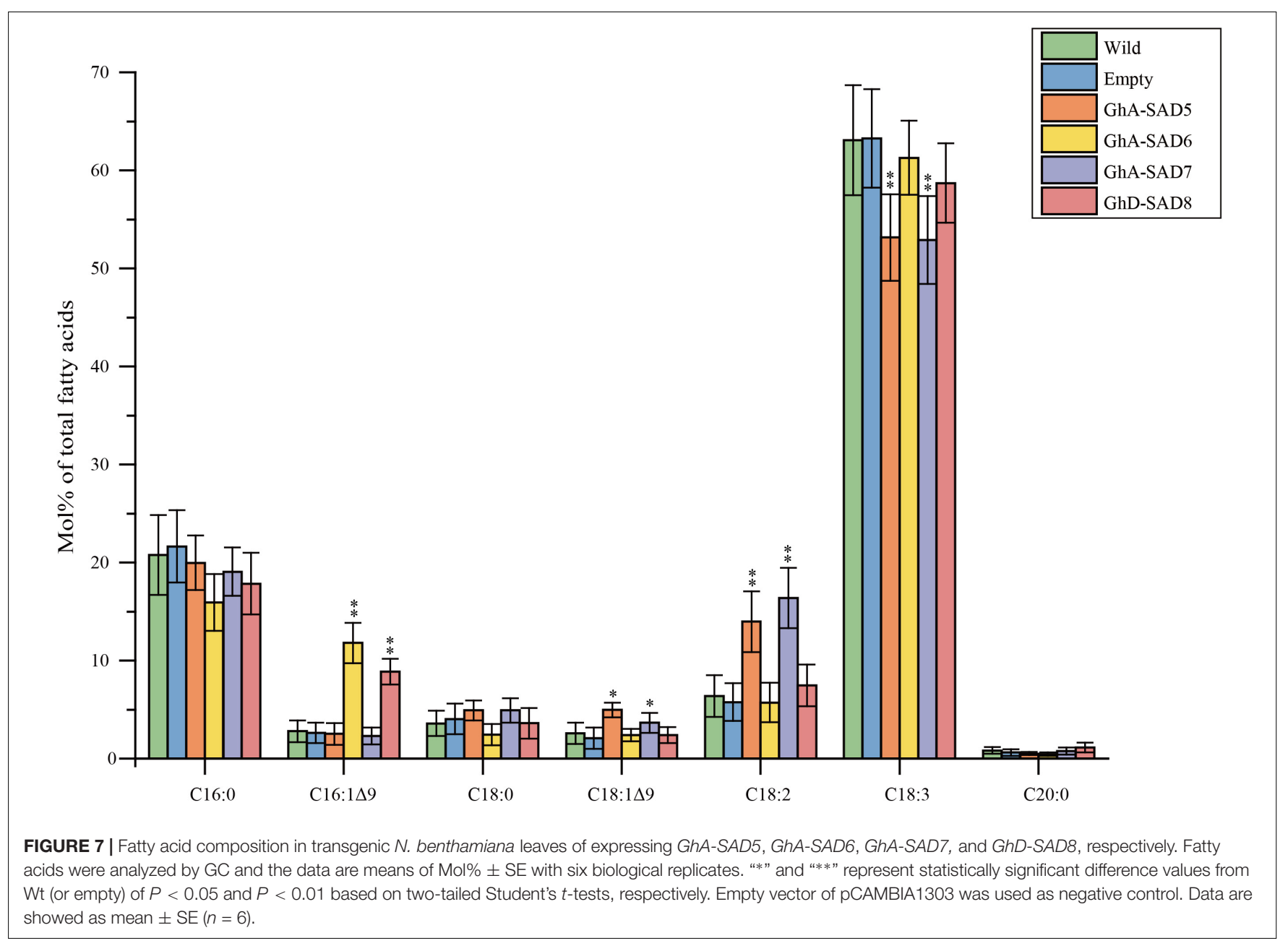

decrease of mature seed dry weight. This indicates that $\omega-7$ FAs may function for seed development. However, seeds from the double mutant of AtAAD3 and AtAAD2 appear normal and can germinate readily under standard growth conditions. Even so, function of $\omega-7$ FAs for seed germination cannot be excluded because the low level of remained $\omega-7$ FAs in the endosperm of this double mutant may impair the examination of possible phenotypes.

To clarify the physiological roles of $\omega-7$ FAs enriched in developing cotton endosperm, we conducted an in vitro cultivation of young embryos isolated from early developing seeds (data not shown). Addition of palmitoleic acid into the medium promoted the embryo development, with the embryo growing more health compared to the embryo cultured in the medium without this $\omega-7$ FA. Moreover, this beneficial effect of $\omega-7$ FA addition exhibited much stronger under cold stress. This demonstrates that $\omega-7$ FA accumulation in the developing endosperm may function importantly for cotton seed development. Further lab work such as CRISPR-Cas9 mediated 16:0-ACP specific GhSAD mutation in developing cotton endosperm is under way to investigate the biological functions of $\omega-7$ FA, particularly for seed development and germination.

\section{Members of Cotton GhSAD Family May Function Differentially, With GhA-SAD6 and GhD-SAD8 as the Major Players for Palmitoleic Acid Enrichment in Developing Cotton Endosperm}

Generally, soluble acyl-ACP $\Delta^{9}$ desaturases (AAD) catalyze the formation of first unsaturated fatty acid in FA biosynthesis pathway in plant plastid. As a polygenic family, AAD contains various isoforms. For example, multiple members of AAD family were identified in several plant genomes, with 7 members in Arabidopsis (Kachroo et al., 2007), 8 members in cacao (Theobroma cacao) (Zhang Y. et al., 2015) and 3 members in olive (Olea europaea) (Parvini et al., 2016), respectively. Moreover, different AAD isoforms showed functional diversity and distinct expression patterns. Among 7 AtSAD members, SSI2/FAB is the predominant and the best characterized isoform, with expression in all seed tissues and 18:0-ACP $\Delta^{9}$ desaturation (Lightner et al., 1994) despite it was also active on 16:0-ACP (Kachroo et al., 2007). Microarray analysis by Le et al. (2010) showed that AtAAD2 expression was mainly in the peripheral endosperm, whereas $A t A A D 3$ largely expressed in the chalazal region of the endosperm (Le et al., 2010; Bryant et al., 2016). 


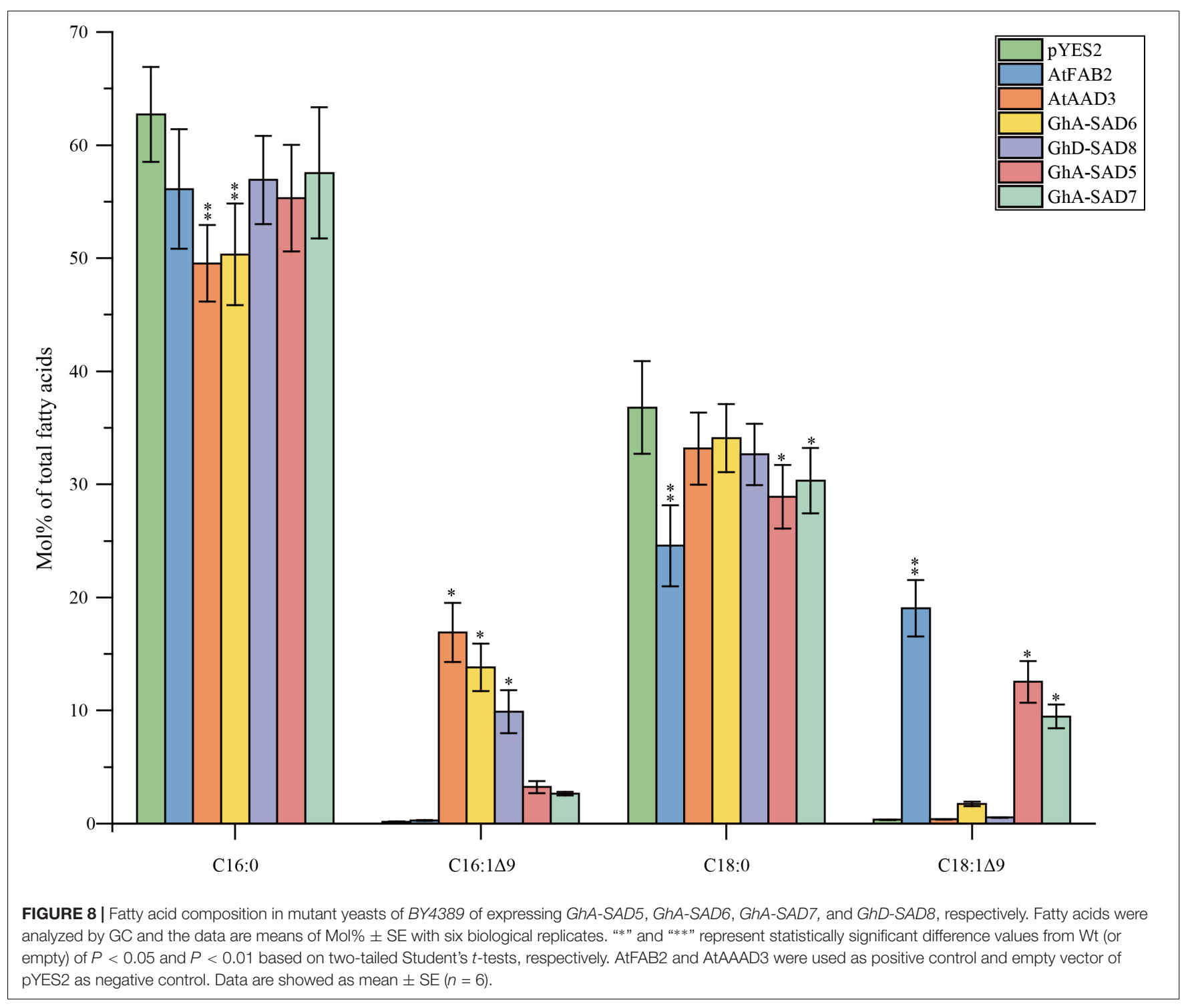

Further analysis on the single or double mutant of the genes by Bryant et al. (2016) evidenced that AtAAD2 and AtAAD3 function in 16:0-ACP $\Delta^{9}$ desaturation and contribute to the biosynthesis of $\omega-7$ FAs specifically in the aleurone. Possibly, the accumulation of $\omega-7$ FAs in rapeseed aleurone plus seed coat results from the homologs of AtAAD3 since rapeseed has the relatively close phylogenetic relationship with Arabidopsis (Bryant et al., 2016). Although such findings are obtained from the characterization of the multigene family of $\mathrm{AAD}$, the functions of most isoforms of this family remain to be elucidated.

Previous study showed that total of 17 stearoyl-ACP $\Delta^{9}$ desaturases (SAD) were identified in upland cotton genome, and their expression were developmentally regulated (Shang et al., 2017). However, no functional analysis of these 17 GhSADs is available, hence raising the question whether these GhSADs are redundant to the monounsaturated fatty acid formation or non-functional enzyme, or function separately from one another.
In the light of the present study, it demonstrates that the identical GhSAD family members were identified in upland cotton (Supplementary Table S3). Four GhSAD members including GhA-SAD5, GhA-SAD6, GhA-SAD7 and GhD-SAD8 showed higher expression levels at early stage of developing ovules than in other vegetative organs (Figure 4). Detailed expression analysis in dissection cottonseeds of different developmental stages revealed that the four genes above were highly expressed in early developing endosperm (15-25 DAF) (Figure 5), especially for GhA-SAD6 and GhD-SAD8 (Figure 5). However, the transcripts of GhA-SAD2, GhA-SAD3, and GhDSAD6 mostly accumulated in the embryo (data not shown). Such diverse spatiotemporal expression patterns indicate that these GhSADs tested may function differentially. In order to investigate their functions, particularly those GhSADs selectively expressed in developing cotton endosperm, we conduct in vivo functional assays including transient expression in tobacco leaves and heterologous expressing in yeast strain BY4389 
unable to synthesize unsaturated fatty acids. The overexpression of both GhA-SAD6 and GhD-SAD8 substantially increase palmitoleic acid $\left(16: 1 \Delta^{9}\right)$ synthesis in leaves, accompanied by a concomitant decrease in palmitic acid (16:0) (Figure 7). Unlike GhA-SAD6 and GhD-SAD8, the transient expression of either GhA-SAD5 or GhA-SAD7 slightly enhance oleic acid $\left(18: 1 \Delta^{9}\right)$ in the leaves at the expense of stearic acid (18:0) (Figure 7). Similar results were also obtained by functional complementation assay in the yeast system (Figure 8). These reveal that in the developing cotton endosperm, GhASAD6 and GhD-SAD8 are responsible for the synthesis of palmitoleic acid $\left(16: 1 \Delta^{9}\right)$ by 16:0-ACP desaturation while GhASAD5 and GhA-SAD7 are responsible for the synthesis of oleic acid $\left(18: 1 \Delta^{9}\right)$ by 18:0-ACP desaturation. Together, our data demonstrate for the first time that the four GhSAD members (GhD-SAD8, GhA-SAD5, GhA-SAD6 and GhA-SAD7) are functional acyl-ACP $\Delta^{9}$ desaturase enzymes but with different substrate preferences. GhA-SAD6 and GhD-SAD8 can preferentially catalyze $16: 0-\mathrm{ACP}$ to $16: 1 \Delta^{9}$-ACP, whereas GhA-SAD5 and GhA-SAD7 are able to selectively catalyze 18:0-ACP to $18: 1 \Delta^{9}$-ACP although in vitro assay has not been conducted yet for identifying the substrate specificity of those GhSAD members. Evolutionally, the occurrence of multiple SAD members in upland cotton and other plant species tested likely provide diverse enzymes with selective expression so as to achieve fine spatiotemporal accumulation of different monounsaturated fatty acids as in cases of the seeds from cotton, Arabidopsis and rapeseed where fatty acid profiles were different between embryo and endosperm. Further functional characterization is required for every member of this SAD family. Nevertheless, our study brings new perspectives in this regard.

\section{Key Amino Acid Residues Lining the Bottom Part of the Substrate-Binding Channels of SADs Are Crucial Determinants of the Substrate Specificity of the Enzymes, but Not the Only Factor}

As described above, plant SAD family composed of soluble plastidial proteins contains multiple members with differential expressions in different tissues and distinct substrate specificities. Some isoforms of the enzyme like castor RcSAD1 and Arabidopsis AtFAB2 show high preference for 18:0-ACP, yielding 18:1 $\Delta^{9}$-ACP, while others such as cat's claw MucPAD, Arabidopsis AtAAD2 and AtAAD3 exhibit strong activity on 16:0-ACP, producing 16:1 $\Delta^{9}$-ACP. It have been reported that several $\Delta^{9}$ desaturases can recognize 14-, 16-, and 18-carbon chain lengths, and others perform desaturation with $\Delta^{6}$ and $\Delta^{9}$ regiospecificities (Whittle et al., 2005; Guy et al., 2007). In view of evolution, such diverse substrate specificities of SADs likely provide a competitive advantage for host plants to produce the required monounsaturated FAs for life activities including maintaining membrane fluidity and channeling into storage triacylglycerols (TAGs) in storage tissues (Shanklin and Cahoon, 1998), benefiting the evolution of novel metabolic diversity (Dyer et al., 2002).

Previous studies on the molecular mechanism underlying SAD substrate specificity were mostly conducted using the crystal structure of castor 18:0-ACP $\Delta^{9}$ desaturase (RcSAD1) as a model (Lindqvist et al., 1996; Cahoon et al., 1997), showing that a group of eight amino acid residues lining the bottom part of the substrate channels of SADs is crucial for determining the substrate specificity of the enzymes (Cahoon et al., 1998). For example, RcSAD1 could change its binding substrate for 16:0ACP from the wild type with 18:0-ACP when two key residues (T117 and G188) were substituted by Arg and Leu. Moreover, the mutant RcSAD1 activity for 16:0-ACP was higher by 82 folds than the wild enzyme for 18:0-ACP (Whittle and Shanklin, 2001). The side chain sizes of these 8 amino acids influence the depth and width of the substrate channel of the enzyme (Cahoon et al., 1997). The substrate channel of the archetype $\Delta^{9}$ SAD is deep enough to accommodate 18:0-acyl chain, thus producing $\Delta^{9}$ monoenes (Figure 2A). However, the shorter substrate channel of the enzyme is more adapted to 16:0-acyl chain, forming a $\omega$-7 FA (e.g., palmitoleic acid, 16:1 $\Delta^{9}$ ) (Figures 2A-C). These eight amino acids appear to be well conserved among the SAD isoforms characterized so far in higher plants. The amino acid sequences of the most SAD isoforms are highly homology to that of the castor 18:0-ACP $\Delta^{9}$ desaturase (RcSAD1), implying that SADs share a common architecture able to accommodate different substrate binding models. Like in Arabidopsis, AtSADs share a high degree of amino acid sequence similarity and a common structural fold (Kachroo et al., 2007). Moreover, the three divergent residues (F226/216, S224/214, and T159/149) of the eight amino acids lining the bottom part of the substrate channels of AtAAD2 and AtAAD3 conferred their strong activity for 16:0-ACP, but not 18:0-ACP catalyzed by the SAD archetype (Bryant et al., 2016).

Base on such conservation, the corresponding amino acid residues in 17 cotton SADs were identified by a polypeptide sequence alignment of the eight amino acid residues lining the bottom part of the substrate channels of the GhSAD proteins using the typical 18:0-ACP specific SADs (RcSAD1 and AtFAB2) and the 16:0-ACP specific SADs (Muc-PAD, AtFAB2, AtAAD2, and AtAAD3) as references (Table 1). Interestingly, the eight amino acids are all identical for the group of 18:0ACP specific SADs. However, they are divergent for the group of 16:0-ACP specific SADs. Only one amino acid residue of L115 in RcSAD1 is identical for all SADs tested here. This alignment indicates that 10 GhSADs may be 18:0-ACP specific while the other seven GhSADs possibly be 16:0-ACP specific. Further 3D modeling of GhSAD protein monomers showed that GhA-SAD6 and GhD-SAD8 had the similar structure of the substrate-bound cavity adjacent to the active diiron center with AtAAD3, but different from RcSAD1, suggesting that these two GhSADs have 16:0-ACP activity. Their substrate specificities were confirmed by our functional assay using tobacco transient expression and yeast expression system (Figures 7, 8). In contrast, GhA-SAD5 and GhA-SAD7 exhibited 18:0ACP preference (Figures 7, 8) although they were classed into the same group of $16: 0-\mathrm{ACP}$ specificity by amino acid 
sequence alignment (Table 1). On the other hand, GhA-SAD6 and GhD-SAD8 only have 50\% identity for these eight amino acid residues despite of the similar substrate specificity, just like cases of Muc-PAD, AtAAD2, and AtAAD3 with less identity but the same substrate activity. Even for RcSAD1 and Muc-PAD, only one residue was diverged for the eight amino acids between them. Taken together, these indicate that other factors may also affect the substrate specificity of SADs beyond the predominant role played by the eight amino acid residues.

In consistence with this, increasing characterization of more SADs then revealed that for substrate preference of a given chain length, some isoforms can also desaturated slightly shorter- or longer-acyl chains, despite of a reduced affinity (Kachroo et al., 2007; Rodriguez et al., 2015). Some AAD specificities were dependent on the substrate chain length. For instance, ivy (Hedera helix) AAD desaturated at the $\Delta^{9}$ position when presented with 18:0-ACP substrate while it catalyzed $\Delta^{4}$ desaturation when supplied with 16:0-ACP (Whittle et al., 2005). Moreover, this AAD also converted 16:1 $\Delta^{9}$-ACP and 18:1 $\Delta^{9}$-ACP to the corresponding $\Delta^{4,9}$ dienes. In contrast to ivy AAD, Thunbergia AAD produced $16: 1 \Delta^{6}$ with $16: 0$ ACP substrate but when presented with 18:0-ACP substrate generated a mixture of $\Delta^{6}$ and $\Delta^{9}$ desaturated products (Cahoon et al., 1997). Possibly, the mechanism determining the substrate specificity of AADs may evolve independently among different plants species.

\section{CONCLUSION}

The present study revealed that palmitoleic acid $\left(16: 1 \Delta^{9}\right)$, a kind of $\omega-7$ FAs, is specifically enriched in developing cotton endosperm, possibly benefiting the embryo development. Total of 17 GhSADs are identified in upland cotton genome and their expressions are spatiotemporally diverse, indicating that they may function differentially. Particularly, GhA-SAD6 and $G h D-S A D 8$ are preferentially expressed in the developing endosperm whereas $G h A-S A D 2$ and $G h D-S A D 6$ are restricted to the embryo. Our functional characterizations using transient expression in tobacco leaves and heterologous expression in yeast mutant BY4389 provide the first evidence showing that GhA-SAD6 and GhD-SAD8 are 16:0-ACP $\Delta^{9}$ desaturases responsible for production of $16: 1 \Delta^{9}$ in the developing cotton endosperm while GhA-SAD5 and GhA-SAD7 act as 18:0-ACP $\Delta^{9}$ desaturases accounting for $18: 1 \Delta^{9}$ biosynthesis in this tissue. In addition, polypeptide sequence alignment and $3 \mathrm{D}$

\section{REFERENCES}

Arnold, K., Bordoli, L., Kopp, J., and Schwede, T. (2006). The SWISS-MODEL workspace: a web-based environment for protein structure homology modelling. Bioinformatics 22, 195-201. doi: 10.1093/bioinformatics/ bti770

Barthole, G., To, A., Marchive, C., Brunaud, V., Soubigou-Taconnat, L., Nathalie, B., et al. (2014). MYB118 represses endosperm maturation in seeds of Arabidopsis. Plant Cell 26, 3519-3537. doi: 10.1105/tpc.114. 130021 modeling of the protein monomers of these GhA-SAD6 and GhD-SAD8 suggested that their 16:0-ACP substrate specificities may be determined by the eight amino acid residues lining the bottom part of their substrate channels which distinguish them from the archetype 18:0-ACP $\Delta^{9}$ desaturase. Taken together, this study brought new insights into the SADmediated biosynthesis and differential accumulation of special lipid reserve in developing cotton seeds. The identified GhASAD6 and GhD-SAD8 could be good endogenous genes for engineering to produce higher oil enriched with $\omega$ 7 FAs in cotton seeds or other oil crops for food and industrial applications.

\section{DATA AVAILABILITY}

No datasets were generated or analyzed for this study.

\section{AUTHOR CONTRIBUTIONS}

BL, JX, XJ, and RL designed the experiments and drafted the manuscript. BL and YS did the vector construction, fatty acid analysis and material plantation. BL and XM carried out the expression and RNA-seq analysis. RL and XJ revised the manuscript.

\section{FUNDING}

This work has been supported by the National Natural Science Foundation of China (Grant No. 31401430), Key Research and Development Project of Shanxi Province (201703D2210023), Introduction of Talent Research Start-up Fund by Shanxi Agricultural University (2014ZZ06), State Ministry of Agriculture "948" Project (2014-Z39), Shanxi Province Key Projects of Coal-based Science and Technology (FT-2014-01), and Research Project Supported by Shanxi Scholarship Council of China (2015-064).

\section{SUPPLEMENTARY MATERIAL}

The Supplementary Material for this article can be found online at: https://www.frontiersin.org/articles/10.3389/fpls.2019.00703/ full\#supplementary-material 
arabidopsis aleurone. Plant Physiol. 172, 154-162. doi: 10.1104/pp.16. 00836

Cahoon, E. B., Lindqvist, Y., Schneider, G., and Shanklin, J. (1997). Redesign of soluble fatty acid desaturases from plants for altered substrate specificity and double bond position. Proc. Natl. Acad. Sci. U.S.A. 94, 4872-4877. doi: 10.1073/ pnas.94.10.4872

Cahoon, E. B., Shah, S., Shanklin, J., and Browse, J. (1998). A determinant of substrate specificity predicted from the acyl-acyl carrier protein desaturase of developing cat's claw seed. Plant Physiol. 117, 593-598. doi: 10.1104/pp.117.2.593

Curb, J. D., Wergowske, G., Dobbs, J. C., Abbott, R. D., and Huang, B. (2000). Serum lipid effects of a high-monounsaturated fat diet based on macadamia nuts. Arch. Intern. Med. 160, 1154-1158. doi: 10.1001/archinte.160. 8.1154

Dyer, J. M., Chapital, D. C., Kuan, J. W., Mullen, R. T., and Pepperman, A. B. (2002). Metabolic engineering of Saccharomyces cerevisiae for production of novel lipid compounds. Appl. Microbiol. Biotechnol. 59, 224-230. doi: 10.1007/ s00253-002-0997-5

Ginter, E., and Simko, V. (2016). New data on harmful effects of trans-fatty acids. Bratisl. Lek. Listy 117, 251-253. doi: 10.4149/BLL_2016_048

Gummeson, P. O., Lenman, M., Lee, M., Singh, S., and Stymne, S. (2000). Characterisation of acyl-ACP desaturases from Macadamia integrifolia Maiden \& Betche and Nerium oleander L. Plant Sci. 154, 53-60. doi: 10.1016/S01689452(99)00268-X

Guy, J. E., Whittle, E., Kumaran, D., Lindqvist, Y., and Shanklin, J. (2007). The crystal structure of the ivy Delta4-16:0-ACP desaturase reveals structural details of the oxidized active site and potential determinants of regioselectivity. J. Biol. Chem. 282, 19863-19871. doi: 10.1074/jbc.M702520200

Huh, J. H., Bauer, M. J., Hsieh, T. F., and Fischer, R. (2007). Endosperm gene imprinting and seed development. Curr. Opin. Genet. Dev. 17, 480-485. doi: 10.1016/j.gde.2007.08.011

Jiang, Y., Guo, W., Zhu, H., Ruan, Y. L., and Zhang, T. (2012). Overexpression of GhSusA1 increases plant biomass and improves cotton fiber yield and quality. Plant Biotechnol. J. 10, 301-312. doi: 10.1111/j.1467-7652.2011.00662.X

Jiao, X., Zhao, X., Zhou, X. R., Green, A. G., Fan, Y., Wang, L., et al. (2013). Comparative transcriptomic analysis of developing cotton cotyledons and embryo axis. PLoS One 8:e71756. doi: 10.1371/journal.pone.0071756

Kachroo, A., Shanklin, J., Whittle, E., Lapchyk, L., Hildebrand, D., Kachroo, P., et al. (2007). The Arabidopsis stearoyl-acyl carrier protein-desaturase family and the contribution of leaf isoforms to oleic acid synthesis. Plant Mol. Biol. 63, 257-271. doi: 10.1007/s11103-006-9086-y

Kallio, H., Yang, B., Peippo, P., Tahvonen, R., and Pan, R. (2002). Triacylglycerols, glycerophospholipids, tocopherols, and tocotrienols in berries and seeds of two subspecies (ssp. sinensis and mongolica) of Sea Buckthorn (Hippophae rhamnoides). J. Agric. Food Chem. 50, 3004-3009. doi: 10.1021/jf 0115560

Le, B. H., Cheng, C., Bui, A. Q., Wagmaister, J. A., Henry, K. F., Pelletier, J., et al. (2010). Global analysis of gene activity during Arabidopsis seed development and identification of seed-specific transcription factors. Proc. Natl. Acad. Sci. U.S.A. 107, 8063-8070. doi: 10.1073/pnas.1003530107

Li, Y., Beisson, F., Pollard, M., and Ohlrogge, J. (2006). Oil content of Arabidopsis seeds: the influence of seed anatomy, light and plant-to-plant variation. Phytochemistry 67, 904-915. doi: 10.1016/j.phytochem.2006.02.015

Lightner, J., Wu, J., and Browse, J. (1994). A mutant of arabidopsis with increased levels of stearic acid. Plant Physiol. 106, 1443-1451. doi: 10.1104/pp.106.4.1443

Lindqvist, Y., Huang, W., Schneider, G., and Shanklin, J. (1996). Crystal structure of delta9 stearoyl-acyl carrier protein desaturase from castor seed and its relationship to other di-iron proteins. EMBO J. 15, 4081-4092. doi: 10.1002/ j.1460-2075.1996.tb00783.x

Liu, Q., Wu, M., Zhang, B., Shrestha, P., Petrie, J., Green, A. G., et al. (2017). Genetic enhancement of palmitic acid accumulation in cotton seed oil through RNAi down-regulation of ghKAS2 encoding beta-ketoacyl-ACP synthase II (KASII). Plant Biotechnol. J. 15, 132-143. doi: 10.1111/pbi.12598

Livak, K. J., and Schmittgen, T. D. (2001). Analysis of relative gene expression data using real-time quantitative PCR and the 2(-Delta Delta C(T)) method. Methods 25, 402-408. doi: 10.1006/meth.2001.1262

McKeon, T. A., and Stumpf, P. K. (1982). Purification and characterization of the stearoyl-acyl carrier protein desaturase and the acyl-acyl carrier protein thioesterase from maturing seeds of safflower. J. Biol. Chem. 257, 12141-12147. doi: $10.1086 / 283964$

Misra, A., Singhal, N., and Khurana, L. (2010). Obesity, the metabolic syndrome, and type 2 diabetes in developing countries: role of dietary fats and oils. J. Am. Coll. Nutr. 29, 289S-301S. doi: 10.1080/07315724.2010.10 719844

Mozaffarian, D., Cao, H., King, I. B., Lemaitre, R. N., Song, X., Siscovick, D. S., et al. (2010). Trans-palmitoleic acid, metabolic risk factors, and new-onset diabetes in U.S. adults: a cohort study. Ann. Intern. Med. 153, 790-799. doi: 10.7326/0003-4819-153-12-201012210-00005

Nguyen, H. T., Mishra, G., Whittle, E., Pidkowich, M. S., Bevan, S. A., Merlo, A. O., et al. (2010). Metabolic engineering of seeds can achieve levels of omega-7 fatty acids comparable with the highest levels found in natural plant sources. Plant Physiol. 154, 1897-1904. doi: 10.1104/pp.110.165340

Nguyen, H. T., Park, H., Koster, K. L., Cahoon, R. E., Nguyen, H. T., Shanklin, J., et al. (2015). Redirection of metabolic flux for high levels of omega-7 monounsaturated fatty acid accumulation in camelina seeds. Plant Biotechnol. J. 13, 38-50. doi: 10.1111/pbi.12233

Parvini, F., Sicardo, M. D., Hosseini-Mazinani, M., Martinez-Rivas, J. M., and Hernandez, M. L. (2016). Transcriptional analysis of stearoyl-acyl carrier protein desaturase genes from olive (Olea europaea) in relation to the oleic acid content of the virgin olive oil. J. Agric. Food Chem. 64, 7770-7781. doi: 10.1021 /acs.jafc.6b02963

Penfield, S., Rylott, E. L., Gilday, A. D., Graham, S., Larson, T. R., Graham, I. A., et al. (2004). Reserve mobilization in the Arabidopsis endosperm fuels hypocotyl elongation in the dark, is independent of abscisic acid, and requires phosphoenolpyruvate carboxykinase1. Plant Cell 16, 2705-2718. doi: 10.1105/ tpc.104.024711

Rodriguez, M. F., Sanchez-Garcia, A., Salas, J. J., Garces, R., and Martinez-Force, E. (2015). Characterization of soluble acyl-ACP desaturases from Camelina sativa, Macadamia tetraphylla and Dolichandra unguis-cati. J. Plant Physiol. 178, 35-42. doi: 10.1016/j.jplph.2015.01.013

Shang, X., Chai, Q., Zhang, Q., Jiang, J., Zhang, T., Guo, W., et al. (2015). Down-regulation of the cotton endo-1,4-beta-glucanase gene KOR1 disrupts endosperm cellularization, delays embryo development, and reduces early seedling vigour. J. Exp. Bot. 66, 3071-3083. doi: 10.1093/jxb/ erv111

Shang, X., Cheng, C., Ding, J., and Guo, W. (2017). Identification of candidate genes from the SAD gene family in cotton for determination of cottonseed oil composition. Mol. Genet. Genomics 292, 173-186. doi: 10.1007/s00438-0161265-1

Shanklin, J., and Cahoon, E. B. (1998). Desaturation and related modifications of fatty acids1. Annu. Rev. Plant Physiol. Plant Mol. Biol. 49, 611-641. doi: 10.1146/annurev.arplant.49.1.611

Song, F., Cui, C. J., Chen, L., Sun, Y. L., Wang, F. F., Hussain, J., et al. (2012). Isolation and characterization of an endosperm-specific promoter from wheat (Triticum aestivum L.). Z. Naturforsch. C 67, 611-619. doi: 10.1515/znc-201211-1212

Sturtevant, D., Horn, P., Kennedy, C., Hinze, L., Percy, R., Chapman, K., et al. (2017). Lipid metabolites in seeds of diverse Gossypium accessions: molecular identification of a high oleic mutant allele. Planta 245, 595-610. doi: 10.1007/ s00425-016-2630-3

Troncoso-Ponce, M. A., Barthole, G., Tremblais, G., To, A., Miquel, M., Lepiniec, L., et al. (2016). Transcriptional activation of two delta-9 palmitoyl-ACP desaturase genes by myb115 and myb118 is critical for biosynthesis of omega-7 monounsaturated fatty acids in the endosperm of Arabidopsis seeds. Plant Cell 28, 2666-2682. doi: 10.1105/tpc.16.00612

Whittle, E., Cahoon, E. B., Subrahmanyam, S., and Shanklin, J. (2005). A multifunctional acyl-acyl carrier protein desaturase from Hedera helix L. (English ivy) can synthesize 16- and 18-carbon monoene and diene products. J. Biol. Chem. 280, 28169-28176. doi: 10.1074/jbc.M504 205200

Whittle, E., and Shanklin, J. (2001). Engineering delta 9-16:0-acyl carrier protein (ACP) desaturase specificity based on combinatorial saturation mutagenesis and logical redesign of the castor delta 9-18:0-ACP desaturase. J. Biol. Chem. 276, 21500-21505. doi: 10.1074/jbc.M102129200

Wu, Y., Li, R., and Hildebrand, D. F. (2012). Biosynthesis and metabolic engineering of palmitoleate production, an important contributor to human 
health and sustainable industry. Prog. Lipid Res. 51, 340-349. doi: 10.1016/j. plipres.2012.05.001

Xue, W. B., Liu, F., Sun, Z., and Zhou, Z. G. (2016). A delta-9 fatty acid desaturase gene in the microalga Myrmecia incisa Reisigl: cloning and functional analysis. Int. J. Mol. Sci. 17:1143. doi: 10.3390/ijms17071143

Yan, D., Duermeyer, L., Leoveanu, C., and Nambara, E. (2014). The functions of the endosperm during seed germination. Plant Cell Physiol. 55, 1521-1533. doi: $10.1093 / \mathrm{pcp} / \mathrm{pcu} 089$

Zhang, J., Li, J., Garcia-Ruiz, H., Bates, P. D., Mirkov, T. E., Wang, X., et al. (2014). A stearoyl-acyl carrier protein desaturase, NbSACPD-C, is critical for ovule development in Nicotiana benthamiana. Plant J. 80, 489-502. doi: 10.1111/tpj. 12649

Zhang, L. F., Li, W. F., Han, S. Y., Yang, W. H., and Qi, L. W. (2013). cDNA cloning, genomic organization and expression analysis during somatic embryogenesis of the translationally controlled tumor protein (tctp) gene from japanese larch (larix leptolepis). Gene 529, 150-158. doi: 10.1016/j.gene.2013.07.076

Zhang, T., Hu, Y., Jiang, W., Fang, L., Guan, X., Chen, J., et al. (2015). Sequencing of allotetraploid cotton (Gossypium hirsutum L. acc. TM-1) provides a resource for fiber improvement. Nat. Biotechnol. 33, 531-537. doi: 10.1038/nbt.3207
Zhang, Y., Maximova, S. N., and Guiltinan, M. J. (2015). Characterization of a stearoyl-acyl carrier protein desaturase gene family from chocolate tree, Theobroma cacao L. Front. Plant Sci. 6:239. doi: 10.3389/fpls.2015. 00239

Zhao, Y., Wang, Y., Huang, Y., Cui, Y., and Hua, J. (2018). Gene network of oil accumulation reveals expression profiles in developing embryos and fatty acid composition in Upland cotton. J. Plant Physiol. 228, 101-112. doi: 10.1016/j. jplph.2018.06.002

Conflict of Interest Statement: The authors declare that the research was conducted in the absence of any commercial or financial relationships that could be construed as a potential conflict of interest.

Copyright (c) $2019 \mathrm{Liu}$, Sun, Xue, Mao, Jia and Li. This is an open-access article distributed under the terms of the Creative Commons Attribution License (CC BY). The use, distribution or reproduction in other forums is permitted, provided the original author(s) and the copyright owner(s) are credited and that the original publication in this journal is cited, in accordance with accepted academic practice. No use, distribution or reproduction is permitted which does not comply with these terms. 